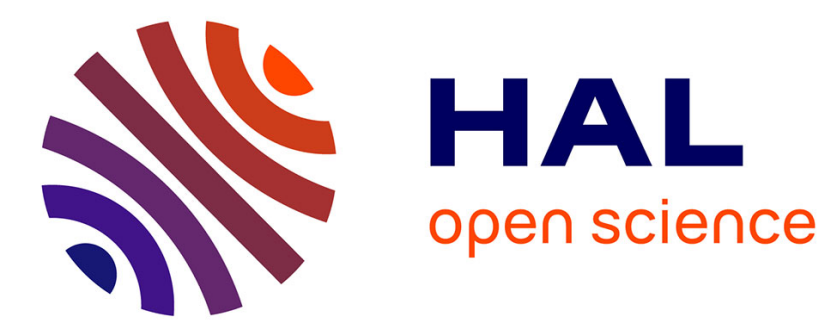

\title{
Classification des séries discrètes pour certains groupes classiques p-adiques
}

Colette Moeglin

\section{To cite this version:}

Colette Moeglin. Classification des séries discrètes pour certains groupes classiques p-adiques. 2006. hal-00017546

\section{HAL Id: hal-00017546 \\ https://hal.science/hal-00017546}

Preprint submitted on 24 Jan 2006

HAL is a multi-disciplinary open access archive for the deposit and dissemination of scientific research documents, whether they are published or not. The documents may come from teaching and research institutions in France or abroad, or from public or private research centers.
L'archive ouverte pluridisciplinaire HAL, est destinée au dépôt et à la diffusion de documents scientifiques de niveau recherche, publiés ou non, émanant des établissements d'enseignement et de recherche français ou étrangers, des laboratoires publics ou privés. 


\title{
Classification des séries discrètes pour certains groupes classiques $p$-adiques
}

\author{
C. Møglin
}

En l'honneur de Roger Howe pour son 60e anniversaire

L'article a pour but de montrer que seule l'hypothèse que des lemmes fondamentaux pour des paires bien précises de groupes est suffisante pour avoir les hypothèses de 10] et [1] permettant de classifier les séries discrètes des groupes symplectiques et orthogonaux "impairs" p-adiques. Le point de départ est l'idée d'Arthur exprimée pleinement sous forme de conjecture en [1] et sous forme de résultats modulo des lemmes fondamentaux en [2].

Le corps de base, noté $F$, est un corps $p$-adique; on suppose que $p \neq 2$ car nos références, surtout 19] utilisent cette hypothèse. Il n'intervient pas dans notre propre travail.

On considère les points sur $F$ d'un groupe classique, c'est-à-dire le groupe des automorphismes d'une forme orthogonale ou symplectique sur un $F$-espace vectoriel, $V$, de dimension finie; ici on se limite aux espaces $V$ symplectiques ou orthogonaux de dimension impaire. On note $n$ est le rang du groupe, c'est-à-dire la partie entière, $[\operatorname{dim} V / 2]$ et $G(n)$ ce groupe p-adique. Si $V$ est orthogonal, on ne suppose pas $V$ maximalement déployée pour obtenir le résultat principal toutefois le cas non maximalement déployé s'obtient à la fin à partir du cas maximalement déployé et dans cette introduction on suppose $G(n)$ déployé. On note $G^{*}(n)$ le groupe complexe des automorphismes de la forme duale au sens de Langlands, c'est-à-dire si $V$ est orthogonal de dimension impaire $G^{*}=S p(2 n, \mathbb{C})$, si $V$ est symplectique de dimension paire $G^{*}=S O(2 n+1, \mathbb{C})$. On notera $n^{*}$ l'entier dimension de la représentation naturelle de $G^{*}$; c'est-à-dire que $n^{*}=2 n$ si $G^{*}(n)$ est un groupe symplectique et $2 n+1$ si $G^{*}(n)$ est un groupe orthogonal. Quand un groupe linéaire $G L(m, F)(m \in \mathbb{N})$ est fixé, on note $\theta$ l'automorphisme de ce groupe $g \mapsto J_{m}^{-1}{ }^{t} g^{-1} J_{m}$, où $J_{m}$ est essentiellement la matrice antidiagonale (cf 1.1 ) et $\tilde{G L}(m, F)$ le produit semi-direct de $G L(m, F)$ par le groupe $\{1, \theta\}$.

L'idée de base d'Arthur pour étudier les groupes classiques est que $G(n)$ est un groupe endoscopique principal pour l'ensemble $\tilde{G}\left(n^{*}\right)$ qui est par définition la composante connexe de $\theta$ dans le produit semi-direct $\tilde{G L}\left(n^{*}, F\right)$. C'est le groupe qui permet de faire un transfert entre les objects stables pour $G(n)$ et les objets stables pour $\tilde{G}\left(n^{*}\right)$. Arthur a aussi expliqué comment obtenir un transfert pour les groupes orthogonaux sur un espace de dimension paire; la "seule" différence est que le transfert fait intervenir des facteurs de transfert que l'auteur ne maîtrise pas.

L'hypothèse faite dans ce papier est que le lemme fondamental pour ce couple soit vrai pour tout $m \leq n$, c'est indispensable pour l'énoncé même des résultats. Mais il faut en plus le lemme fondamental pour tous les groupes endoscopiques intervenant pour la stabilisation de $\tilde{G}\left(m^{*}\right)$, pour tout $m \leq n$. Et il y a un résultat d'Arthur qui doit même, je crois, supposer la validité des lemmes fondamentaux comme ci-dessus pour $m>n$; on peut sans doute borner $m$ mais sans doute pas suffisamment pour que des cas particuliers puissent vraiment être démontrés sans que le cas général le soit. On n'a par contre pas besoin de leur analogue pondéré (sauf erreur); on utilise de façon déterminante les résultats locaux de [2] mais pas les résultats globaux.

Pour la détermination des représentations cuspidales et donc la classification complète, on a aussi besoin des lemmes fondamentaux pour tous les groupes endoscopiques elliptiques de $\tilde{G L}\left(n^{*}, F\right)$. Pour cette classification complète, nous nous sommes limités aux groupes $G$ orthogonaux sur un espace de dimension impaire bien que les méthodes soient générales; la raison de cette limitation est que l'on ne veut pas ici discuter le cas des groupes orthogonaux pairs qui méritent une discussion à eux tout seuls. Pour eux, dans tous mes travaux, à la suite d'Adams, j'ai choisi de prendre comme groupe dual un groupe non connexe, le groupe orthgonal complexe; ce n'est pas le choix d'Arthur et il y a donc à écrire les équivalences entre les 2 points de vue.

Une représentation dont la classe d'isomorphie est invariante sous l'action de $\theta$ sera dite une représentation $\theta$-invariante. Pour tout $m$, on appelle série $\theta$-dicrète une représentation irréductible tempérée de $G L(m, F), \theta$ invariante et qui n'est induite propre d'aucune représentation $\theta$-invariante d'un sous-groupe de Levi $\theta$-invariant de $G L(m, F)$.

Cette définition est beaucoup plus simple du côté groupe dual; on rappelle que la conjecture de Langlands locale pour les $G L([6]$, [7]) et les résultats de Zelevinsky (21]) permettent d'associer à toute représentation irréductible tempérée de $G L(m, F)$ une classe de conjugaison d'un morphisme continu, borné de $W_{F} \times S L(2, \mathbb{C})$ 
dans $G L(m, \mathbb{C})$. Pour $\pi^{G L}$ une représentation irréductible tempérée de $G L(m, F)$ on note $\psi_{\pi^{G L}}$, ce morphisme ; le fait que $\pi^{G L}$ est $\theta$-invariante est exactement équivalent à ce que (quitte à conjuguer) $\theta \circ \psi_{\pi^{G L}} \circ \theta=\psi_{\pi G L}$, où ici $\theta$ est l'automorphisme $g \mapsto{ }^{t} g^{-1}$ de $G L(m, \mathbb{C})$. Le fait que $\pi^{G L}$ est $\theta$-discrète est équivalent à ce que le sous-groupe du centralisateur de $\psi_{\pi G L}$ dans $G L(m, \mathbb{C})$ formé par les éléments $\theta$-invariants est fini.

Soit encore $m$ un entier et soit $\pi^{G L}$ une représentation tempérée de $G L\left(m^{*}, F\right), \theta$-invariante; on fixe un prolongement de $\pi^{G L}$ à $\tilde{G L}\left(m^{*}, F\right)$. Le caractère de cette représentation restreint à $\tilde{G}\left(n^{*}\right)$ n'est pas nécessairement stable. C'est même un résultat profond de 20 30.1 de préciser que ce caractère est stable précisément quand le morphisme associé se factorise par $G^{*}(n)$; c'est pour pouvoir utiliser ce résultat que l'on a supposé la totalité des lemmes fondamentaux. Et en suivant Arthur, quand le caractère de $\pi^{G L}$ vue comme distribution sur $\tilde{G}\left(n^{*}\right)$ est stable, on définit le paquet de $\pi^{G L}$, noté $\Pi\left(\pi^{G L}\right)$ comme étant l'ensemble des représentations irréductibles, $\pi$ de $G(m)$ tel que pour un bon choix de coefficients $c_{\pi} \in \mathbb{R}_{\geq 0}$ la distribution $\operatorname{tr} \pi^{G L}$ de $G L\left(m^{*}, F\right) \times \theta$ soit un transfert de $\sum_{\pi \in \Pi\left(\pi^{G L}\right)} c_{\pi} t r \pi$ ( $\left.\mathrm{cf} 1.3\right)$; cette condition définit uniquement les éléments de $\Pi\left(\pi^{G L}\right)$. Si $\psi$ est le morphisme correspondant à $\pi^{G L}$, on note $\Pi(\psi)$ au lieu de $\Pi\left(\pi^{G L}\right)$. Comme Arthur a en vue des résultats globaux, il prolonge cette définition pour toute représentation $\pi^{G L}$ composante locale d'une forme automorphe cuspidale de $G L\left(m^{*}\right)$; la généralisation est évidente, c'est une induction, il prolonge d'ailleurs aussi les constructions pour obtenir toutes les formes automorphes de carré intégrable mais nous n'en avons pas besoin ici. Une remarque par exemple dans 19 VI.1 (ii) est que si $\pi^{G L}$ est $\theta$-discrète alors $\Pi\left(\pi^{G L}\right)$ est formé de représentations elliptiques ; comme les coefficients $c_{\pi}$ sont positifs d'après Arthur, on peut encore remplacer elliptiques par séries discrètes. Toutefois, on redémontrera ce dont on a besoin pour éviter cet argument croisé.

Pour pouvoir utiliser ce qui précède, la remarque à vérifier est que si $\pi$ est une série discrète de $G(n)$ irréductible alors le caractère distribution de cette représentation appartient à un paquet stable, c'est-à-dire qu'il existe $\pi^{G L}, \theta$-discret comme ci-dessus tel que $\pi \in \Pi\left(\pi^{G L}\right)$; cela est fait en 1.3 . Ensuite, il n'est pas difficile de vérifier que cela entraîne que le support cuspidal de $\pi$ est "demi-entier" (cf. 1.4) et par voie de conséquence, cela nécessite que les points de réductibilité des induites de cuspidales soient demi-entiers (cf. 2). Ceci est une partie des hypothèses de [10] et [11] et on peut aller plus loin pour avoir toutes les hypothèses. Ainsi on associe à $\pi$ le morphisme $\psi_{\pi^{G L}}$ tel que $\pi \in \Pi\left(\psi_{\pi^{G L}}\right)$. A ce point, il me semble que l'unicité de $\pi^{G L}$ n'est pas complètement claire; on (re)trouvera cette unicité de façon détournée à la fin du travail en 3.

On peut faire cette construction pour $\pi_{0}$ une représentation cuspidale de $G(n)$ et donc obtenir un morphisme $\psi_{0}$ de $W_{F} \times S L(2, \mathbb{C})$ dans $G L\left(n^{*}, \mathbb{C}\right)$. Ce morphisme détermine et est déterminé par les propriétés d'irréductibilité des induites de la forme $S t(\rho, a) \times \pi_{0}$, où $\rho$ est une représentation irréductible, cuspidale autoduale d'un groupe $G L\left(d_{\rho}, F\right)$ et $a$ est un entier; $S t(\rho, a)$ est la représentation de Steinberg généralisée de $G L\left(a d_{\rho}, F\right)$, l'induite étant une représentation de $G\left(n+a d_{\rho}\right)$. Ce lien se fait exactement comme conjecturé en [10] et [11] :

on voit $\psi_{0}$ comme une représentation de $W_{F} \times S L(2, \mathbb{C})$ dans $G L\left(n_{0}^{*}, \mathbb{C}\right)$. Toute représentation irréductible de $W_{F} \times S L(2, \mathbb{C})$ est le produit tensoriel d'une représentation irréductible, notée $\rho$, de $W_{F}$, et d'une représentation de dimension finie $\sigma_{a}$ de $S L(2, \mathbb{C})$ uniquement déterminée par sa dimension notée ici $a \in \mathbb{N}$; on note $d_{\rho}$ la dimension de la représentation $\rho$ et on identifie $\rho$ à une représentation cuspidale irréductible de $G L\left(d_{\rho}, F\right)$ grâce à la correspondance locale de Langlands démontrée en [6], [7] et on garde la même notation $\rho$. Et on montre qu'une telle représentation irréductible $\rho \otimes \sigma_{a}$ de $W_{F} \times S L(2, \mathbb{C})$ intervient dans $\psi_{0}$ si et seulement si les 2 conditions suivantes sont réalisées :

(1) $\rho \otimes \sigma_{a}$ à conjugaison près est à valeurs dans un groupe de même type que $G^{*}$, c'est-à-dire orthogonal si ce groupe est orthogonal et symplectique sinon;

(2) l'induite pour le groupe $G\left(n_{0}+d_{\rho}\right), S t(\rho, a) \times \pi_{0}$ est irréductible, où $S t(\rho, a)$ est la représentation de Steinberg basée sur $\rho$ du groupe $G L\left(a d_{\rho}, F\right)$.

Et on montre aussi que si $\rho \otimes \sigma_{a}$ intervient comme sous-représentation de $\psi_{0}$, elle y intervient avec multiplicité 1.

Ces propriétés entraînent l'unicité de $\psi_{0}$ puisque l'on connaît sa décomposition en représentations irréductibles grâce à des propriétés de la représentation $\pi_{0}$; c'est ce que l'on a appelé la connaissance des blocs de Jordan de $\pi_{0}$ et de $\psi_{0}$.

Le lien avec les points de réductibilité des induites de la forme $\rho||^{x} \times \pi_{0}$, où $\rho$ est comme ci-dessus et $x$ est un nombre réel, est le suivant. Pour $\rho$ fixée vue comme une représentation irréductible de $W_{F}$ de dimension $d_{\rho}$, on note $\operatorname{Jord}_{\rho}\left(\psi_{0}\right)$ l'ensemble des entiers a (s'il en existe) tel que $\rho \otimes \sigma_{a}$ soit une sous-représentation irréductible de $\psi_{0}$. Si $\operatorname{Jord}_{\rho}\left(\psi_{0}\right) \neq \emptyset$, on note alors $a_{\rho, \psi_{0}}$ son élément maximal. Supposons maintenant que $\operatorname{Jord}_{\rho}\left(\psi_{0}\right)=\emptyset$; si $\rho \otimes \sigma_{2}$ est à valeurs dans un groupe de même type que $G^{*}$ (cf. ci-dessus); on pose $a_{\rho, \psi_{0}}$ et sinon c'est $\rho$ qui est à valeurs dans un groupe de même type que $G^{*}$ et on pose $a_{\rho, \psi_{0}}:=-1$.

On voit maintenant $\rho$ comme une représentation cuspidale irréductible de $G L\left(d_{\rho}, F\right)$. On définit $x_{\rho, \pi_{0}}$ comme l'unique d'après [16] réel positif ou nul tel que l'induite $\left.\rho\right|^{x_{\rho, \pi_{0}}} \times \pi_{0}$ soit réductible et on montre que (cf. 2, 
ci-dessous) que $x_{\rho, \pi_{0}}=\left(a_{\rho, \psi_{0}}+1\right) / 2$.

Au passage, on démontre que $\psi_{0}$ est sans trou c'est-à-dire que si $\psi_{0}$ contient une représentation de la forme $\rho \otimes \sigma_{a}$ avec $a>2$, alors il contient aussi la représentation $\rho \otimes \sigma_{a-2}$ (ici $\rho$ est une représentation irréductible de $\left.W_{F}\right)$. Pour démontrer le résultat annoncé calculant les points de réductibilité, on démontre d'abord que $x_{\rho, \pi_{0}}$ est un demi-entier, puis on vérifie tous les cas sauf le dernier cas ci-dessus, en utilisant la compatibilité des paquets à l'induction et la restriction (cela a déjà été fait en [13]). Puis on montre que le dernier cas en résulte "par défaut".

Avec ces résultats sur les représentations cuspidales, [10] et [11] associent à toute série discrète irréductible un morphisme de $W_{F} \times S L(2, \mathbb{C})$ dans $G L\left(n^{*}, \mathbb{C}\right)$, noté $\psi_{\pi}$, il n'y a plus qu'à démontrer la compatibilité avec les résultats d'Arthur, c'est-à-dire que $\pi \in \Pi\left(\psi_{\pi}\right)$; c'est fait en 3 .

Dans la suite du travail, on se limite comme expliqué ci-dessus au cas où $G=S O(2 n+1, F)$ (déployé ou non). On suppose que tous les lemmes fondamentaux pour les groupes endoscopiques de $S O(2 m+1, F)$ et pour $\tilde{G L}(2 m, F)$ sont vérifiés pour tout $m$. Il y a une autre propriété dont on a besoin qui peut s'exprimer très facilement ainsi : avec la notation $\Pi(\psi)$ déjà employée, il n'y a qu'une combinaison linéaire (à homothétie près) d'éléments de $\Pi(\psi)$ qui est stable et toute combinaison linéaire stable de séries discrètes et dans l'espace vectoriel engendré par ces éléments. On discute cette propriété en 4.3 car il est vraisemblable qu'elle est dans (ou puisse être démontré par les méthodes de) [2] ; mais comme elle n'est pas écrite telle quelle, on la prend comme hypothèse. Tel que nous avons écrit les arguments, nous avons besoin de cette hypothèse pour tous les morphismes $\psi^{\prime}$ à valeurs dans $S p(2 m, \mathbb{C})$ pour tout $m \leq n$. Avec les lemmes fondamentaux et cette hypothèse, on montre que l'application définie par Arthur de $\Pi(\psi)$ dans l'ensemble des caractères du groupe $\operatorname{Cent}_{S p(2 n, \mathbb{C})} \psi / \operatorname{Cent}(\operatorname{Sp}(2 n, \mathbb{C}))$ est une bijection; un tel résultat est propre au cas des paquets tempérés. On obtient alors la preuve de notre conjecture sur la classification des représentations cuspidales de $S O(2 m+1, F)$ (pour tout $m \leq n$ ), à savoir cet ensemble de représentations cuspidales est en bijection avec l'ensemble des couples, $\psi, \epsilon$, formé d'un morphisme $\psi$ sans trou de $W_{F} \times S L(2, \mathbb{C})$ dans $S p(2 m, \mathbb{C})$, définissant une représentation semi-simple sans multiplicité de $W_{F} \times S L(2, \mathbb{C})$ et d'un caractère $\epsilon$ du centralisateur de $\psi$ dans $S p(2 n, \mathbb{C})$, trivial sur le centre de $S p(2 n, \mathbb{C})$, qui a les propriétés suivantes :

$\epsilon$ est alterné (ou cuspidale au sens de Lusztig) c'est-à-dire : soit une sous-représentation irréductible $\rho \otimes \sigma_{a}$ de $W_{F} \times S L(2, \mathbb{C})$ de dimension $a d_{\rho}$ intervenant dans $\psi$; elle est nécessairement à valeurs dans un groupe $S p\left(a d_{\rho}, \mathbb{C}\right)$ dont on note $Z_{\rho \otimes \sigma_{a}}$ le centre, sous-groupe à 2 éléments qui est naturellement un sous-groupe du centralisateur de $\psi$. Alors $\epsilon$ est alterné si pour $\rho, a$ comme ci-dessus, la restriction de $\epsilon$ à $Z_{\rho \otimes \sigma_{a}} \simeq\{ \pm 1\}$ est non trivial si $a=2$ et si $a>2$, cette restriction n'est pas le même caractère que la restriction de $\epsilon$ à $Z_{\rho \otimes \sigma_{a-2}} \simeq\{ \pm 1\}$;

On remarque que pour cette conjecture précise, on n'a pas traité le cas non déployé qui devrait pourtant être analogue en remplaçant la condition sur la restriction du caractère au centre de $S p(2 n, \mathbb{C})$ par son opposé.

C'est la dernière conjecture qui manquait pour pouvoir décrire complètement les paquets de Langlands de série discrète suivant [10] et [11]; on vérifie pour finir que nos constructions sont bien compatibles avec celles d'Arthur. Il s'agit ici de vérifier que si $\pi \in \Pi(\psi)$, le caractère associé par Arthur se lit sur le module de Jacquet de $\pi$. On montre donc la propriété suivante, soit comme ci-dessus $\rho \otimes \sigma_{a}$ une sous-représentation irréductible de $W_{F} \times S L(2, \mathbb{C})$ incluse dans $\psi$. Supposons d'abord qu'il existe $b \in \mathbb{N}$ avec $b<a$ et $\rho \otimes[b]$ une sous-représentation de $\psi$; on note alors $a_{-}$le plus grand entier $b$ vérifiant ces 2 conditions. Quand un tel $b$ n'existe pas $a_{-}$n'est pas défini si $a$ est impair et vaut 0 si $a$ est pair. On note encore $\rho$ la représentation cuspidale de $G L\left(d_{\rho}, F\right)$ correspondant à $\rho$ par la correspondance de Langlands locale pour $G L\left(d_{\rho}, F\right)$ ([6], 阿). Soit $\pi \in \Pi(\psi)$ et $\epsilon_{\mathcal{A}}(\pi)$ le caractère du centralisateur de $\psi$ associé par Arthur à $\pi$; on montre, avec les notations déjà introduites ci-dessus, que la restriction de $\epsilon_{\mathcal{A}}(\pi)$ à $Z_{\rho \otimes \sigma_{a}}$ est identique à la restriction de ce caractère à $Z_{\rho \otimes \sigma_{a_{-}}}$( est triviale si $a_{-}=0$ ) si et seulement si il existe une représentation $\pi^{\prime}$ du groupe $S O\left(2\left(m-d_{\rho}\left(a-a_{-}\right) / 2\right)+1, F\right)$ et une inclusion :

$$
\pi \hookrightarrow \rho||^{(a-1) / 2} \times \cdots \times \rho \|^{\left(a_{-}+1\right) / 2} \times \pi^{\prime} .
$$

Je remercie Laurent Clozel pour ces explications sur la globalisation de situations locales et Jean-Loup Waldspurger dont les travaux sont indispensables à cet article.

L'essentiel des idées encore développées ici et que j'utilise depuis de nombreuses années m'ont été inspirées par l'étude de la correspondance de Howe (avec l'interprétation donnée par J. Adams). C'est donc un immense plaisir pour moi que de pouvoir dédier cette article à Roger Howe pour son soixantième anniversaire. Je remercie aussi les organisateurs du congrès en son honneur pour leur accueil extrêmement chaleureux. 


\section{Support cuspidal des séries discrètes}

\section{$1.1 \quad$ Notations}

Dans tout l'article sauf la partie 5, si $G(n)$ est un groupe orthogonal, on le suppose déployé. On reprend les notations de l'introduction, pour tout $m \in \mathbb{N}, G(m), G^{*}(m), m^{*}$. Et pour tout $m$, on note $J$ la matrice

$$
\left(\begin{array}{ccc}
0 & \cdots & 1 \\
\vdots & \cdots & \vdots \\
(-1)^{m-1} & \cdots & 0
\end{array}\right)
$$

de façon à pouvoir définir l'action de $\theta$ sur $G L(m, F)$ par $g \mapsto J^{t} g^{-1} J^{-1}$ pour tout $g \in G L(m, F)$.

\subsection{Quelques rappels des résultats de [2]}

On rappelle des résultats fondamentaux pour nous de [2] 30.1; soit $\psi$ un morphisme continu borné de $W_{F} \times S L(2, \mathbb{C})$ dans $G^{*}(n)$. On le voit comme un morphisme de $W_{F} \times S L(2, \mathbb{C}) \times S L(2, \mathbb{C})$ dans $G^{*}(n)$ trivial sur la deuxième copie de $S L(2, \mathbb{C})$. Arthur a défini une action de $\theta$ sur la représentation $\pi(\psi)$ de $G L\left(n^{*}, F\right)$ en imposant à $\theta$ d'induire l'action triviale sur l'espace des fonctionnelles de Whittaker (ceci nécessite a priori un choix d'un caractère additif mais on renvoit à [13] pour l'indépendance et une discussion plus générale); pour donner un sens précis à cela, il faut fixer, à l'aide du caractère addifif fixé, un caractère du groupe des matrices unipotentes supérieures invariant par $\theta$; le module des co-invariants de $\pi(\psi)$ pour ce groupe unipotent et ce caractère est de dimension 1 et l'action de $\theta$ est fixée de telle sorte que $\theta$ agisse trivialement dans cet espace de co-invariants. Ceci dit, pour nous ici, la description de la normalisation n'a acune importance.

Arthur en loc. cit. montre alors l'existence d'un paquet fini de représentations $\Pi(\psi)$ de $G(n)$ et de coefficients entiers positifs $c_{\pi}$ tel que $\operatorname{tr} \pi(\psi) \circ \theta$ soit un transfert stable de la distribution $\sum_{\pi \in \Pi(\psi)} c_{\pi} \operatorname{tr} \pi$; les propriétés des coefficients sont cachés dans la définition de $\tilde{\Pi}_{\text {fin }}$ d'Arthur, ils ne joueront pas de rôle ici sauf la positivité (à un signe commun à tous les coefficients près)! Les représentations incluses dans $\Pi(\psi)$ sont uniquement déterminées grâce à l'indépendance linéaire des caractères. On dit que le morphisme $\psi$ est $\theta$-discret si vu comme une représentation de $W_{F} \times S L(2, \mathbb{C})$ dans $G L\left(n^{*}, \mathbb{C}\right)$, il définit une représentation sans multiplicité de ce groupe; c'est exactement la même définition que celle de l'introduction.

\subsection{Appartenance à un paquet stable}

Soit $\pi$ une série discrète irréductible de $G(n)$. Il faut savoir que la projection du caractère distribution de $\pi$ sur l'ensemble des distributions stables à support dans les éléments elliptiques est non nulle. C'est un résultat local et on va en donner 2 démonstrations.

\subsection{1 argument global}

La première démonstration repose sur la globalisation suivante qui m'a été indiquée par Laurent Clozel et qui se trouve dans [5] theorem $1 \mathrm{~B}, \pi$ peut se globaliser, c'est-à-dire être considérée comme une composante locale d'une forme automorphe de carré intégrable $\Sigma$ d'un groupe adélique associé à $G$; on peut imposer à $\Sigma$ ce que l'on veut en un nombre fini de places, par exemple d'être cuspidale en une autre place et Steinberg en au moins 2 autres places. Cela assure que $\Sigma$ est cuspidale et qu'elle intervient dans une formule des traces simple automatiquement stable; on peut alors d'après [2] 30.2 (b) trouver une forme automorphe $\tilde{\Sigma}$ du groupe adélique associé à $G L\left(n^{*}\right)$ tel que $\pi$ soit dans le paquet associé par Arthur à la composante locale de $\tilde{\Sigma}$ pour notre place; mais Arthur assure même qu'en toute place la composante de $\Sigma$ est dans un paquet stable dont un transfert est donnée par la composante en cette même place de $\Sigma$. On a supposé que $\Sigma$ est Steinberg en au moins une place mais une telle représentation est automatiquement stable et son seul transfert possible parmi les composantes locales de forme automorphe de carré intégrable est la représentation de Steinberg; on peut le vérifier avec des arguments de module de Jacquet, ici on insiste sur le fait que le transfert se définit par des égalités sur toutes les classes de conjugaisons stables pas seulement les elliptiques. Ainsi $\tilde{\Sigma}$ est nécessairement une représentation automorphe cuspidal. Et la composante locale de $\tilde{\Sigma}$ en la place qui nous intéresse, est une représentation notée $\tilde{\pi}$ de $G L\left(n^{*}, F\right)$ telle que $\pi \in \Pi(\tilde{\pi})$; on sait que $\tilde{\pi}$ est $\theta$-stable mais même plus qu'elle provient du groupe endoscopique $G(n)$, c'est un des résultats de [2] 30.2. On ne connaît pas la conjecture de Ramanujan, on écrit donc $\tilde{\pi}$ comme une induite de la forme

$$
\times_{(\rho, a, x) \in \mathcal{I}} S t(\rho, a)||^{x} \times \tilde{\pi}_{0} \times S t(\rho, a)||^{-x},
$$


où $\mathcal{I}$ est un ensemble d'indices paramétrant des triplets formés d'une représentation cuspidale unitaire irréductible $\rho$ d'un $G L\left(d_{\rho}, F\right)$, d'un entier $a$ et d'un réel $\left.x \in\right] 0,1 / 2$ [ et où $\tilde{\pi}_{0}$ est tempérée et son paramètre est à valeurs (à conjugaison près) dans un groupe $G^{*}\left(n_{0}\right)$ pour $n_{0}$ convenable. On pose $\sigma:=\times_{(\rho, a, x) \in \mathcal{I}} S t(\rho, a)||^{x}$ et la $\theta$-stabilité entraîne que

$$
\theta(\sigma)=\times_{(\rho, a, x) \in \mathcal{I}} S t(\rho, a)||^{-x} .
$$

A $\tilde{\pi_{0}}$, on associe en suivant Arthur 30.1 un paquet de représentations $\Pi\left(\tilde{\pi}_{0}\right)$ de $G\left(n_{0}\right)$ où $n_{0}$ est convenable comme ci-dessus. Pour des coefficients $c_{\pi_{0}} \in \mathbb{R}$ positifs on a une égalité de

$$
\sum_{\pi_{0} \in \Pi_{0}} c_{\pi_{0}} \operatorname{tr} \pi_{0}\left(h^{\prime}\right)=\operatorname{tr} \tilde{\pi}_{0}\left(g^{\prime}, \theta\right)
$$

pour tout $h^{\prime}$ suffisamment régulier dans $G\left(n_{0}\right)$, où $g^{\prime}, \theta$ a une classe de conjugaison stable qui correspond, dans le transfert, à la classe de conjugaison stable de $h^{\prime}$ (cf [19] I.3, III.1, III.2). Les formules explicites pour le calcul de la trace d'une induite donne ici avec les notations de (1) :

$$
\operatorname{tr}\left(\sigma \times \tilde{\pi}_{0} \times \theta(\sigma)\right)(g, \theta)=\sum_{\pi_{0} \in \Pi\left(\tilde{\pi}_{0}\right)} c_{\pi_{0}} \operatorname{tr}\left(\sigma \times \pi_{0}\right)(h)
$$

quand la classe de conjugaison stable de $h$ et celle de $(g, \theta)$ se correspondent. Ainsi la représentation $\pi$ est un sous-quotient irréductible de l'une des induites $\sigma \times \pi_{0}$ pour $\pi_{0} \in \Pi\left(\pi_{0}\right)$. Remarquons aussi pour la suite que si $\psi_{0}$ n'est pas $\theta$ discret alors il existe $\psi_{0}^{\prime}$ une représentation de $W_{F} \times S L(2, \mathbb{C})$ dans $G L\left(m_{0}^{*}\right.$, $\left.\mathbb{C}\right)$ se factorisant par $G^{*}\left(m_{0}\right), m_{0}$ un entier convenable et une représentation irréductible $\rho \otimes \sigma_{a}$ de $W_{F} \times S L(2, \mathbb{C})$ tels que

$$
\psi_{0}=\psi_{0}^{\prime} \oplus\left(\rho \otimes \sigma_{a}\right) \oplus\left(\theta(\rho) \otimes \sigma_{a}\right) .
$$

Et, comme ci-dessus, on peut écrire de façon imagée

$$
\Pi\left(\psi_{0}\right)=S t(\rho, a) \times \Pi\left(\psi_{0}^{\prime}\right),
$$

ce qui veut dire que les éléments du membre de gauche sont tous les sous-quotients irréductibles du membre de droite, c'est-à-dire des induites $S t(\rho, a) \times \pi_{0}^{\prime}$ avec $\pi_{0}^{\prime} \in \Pi\left(\psi_{0}^{\prime}\right)$. Par construction les éléments de $\Pi\left(\psi_{0}^{\prime}\right)$ sont des représentations unitaires et les induites ci-dessus sont donc semi-simples. Dès que l'on aura démontré que $\sigma$ est nécessairement inexistant, on saura que, puisque $\pi$ est une série discrète, $\psi_{0}$ est $\theta$-discret.

En particulier si $\pi$ est cuspidal, alors $\psi=\psi_{0}$ car $\pi$ ne peut être un sous-quotient de $\sigma \otimes \pi_{0}$ avec $\pi_{0} \in \Pi\left(\psi_{0}\right)$ et donc $\psi_{0}$ est $\theta$-discret.

Le défaut de cet argument est qu'il utilise de façon assez forte la partie globale des résultats d'Arthur et il n'est donc pas clair à priori, qu'il ne faille pas les lemmes fondamentaux pondérés. On va donc donné un argument local.

\subsection{2 argument d'apparence locale}

Ci-dessous tous les arguments sont locaux mais ils utilisent des résultats qui ont été obtenu avec des formules des traces simples, donc des arguments globaux. Ils m'ont été donnés par Waldspurger.

On sait que le caractère distribution d'une série discrète, $\pi$, de $G(n)$, peut se voir comme une distribution sur l'ensemble des classes de conjugaison d'éléments de $G(n)$ dont la restriction aux classes de conjugaison d'éléments elliptiques est non nulle. En évaluant sur ces intégrales orbitales, on définit une application de l'espace vectoriel engendré par ces caractères de représentations dans un espace $I_{c u s p}(G(n))$, l'espace des intégrales orbitales des pseudo-coefficients, étudié par Arthur en [3] ; en élargissant l'espace des caractères à l'ensemble des caractères des représentations elliptiques de $G(n)$ (définition d'Arthur) on obtient ainsi une application bijective. Arthur dans [3] a donné une décomposition en somme directe de cet espace $I_{c u s p}(G(n))$, l'un des facteurs étant l'espace des distributions stables à support dans les éléments elliptiques. On a déjà véfié en [12] que pour définir la projection sur ce facteur stable, seul le lemme fondamentale stable est nécessaire. On utilise la notation $I_{c u s p}^{s t}(G(n))$ pour cet espace, conforme aux notations de [19] VI. 1.

Et le point est de démontrer que la projection du caractère distribution de $\pi$ sur cet espace est non nulle; on le fait en localisant au voisinage de l'origine ce qui permet d'utiliser le développement asymptotique d'HarishChandra d'où une décomposition en somme de transformée de Fourier d'intégrales orbitales unipotentes; la dimension des orbites donnent un degré d'homogénéité pour chacun de ces coefficients. La stabilisation respecte le degré d'homogénéité. On regarde le coefficient relatif à l'orbite triviale, c'est le degré formel de la série discrète et ce coefficient est donc non nul; la transformée de Fourier de l'orbite de l'élément 0 est automatiquement stable et a donc une projection non nulle sur l'analogue de $I_{c u s p}^{s t}(G(n))$ dans l'algèbre de Lie. Ceci est le terme de degré 
0 dans le developpement asymptotique de la projection du caractère de $\pi$ sur $I_{c u s p}^{s t}(G(n))$ d'où la non nullité de cette projection.

On utilise ensuite [19] VI.1 proposition (b) qui démontre que le transfert induit un isomorphisme d'espace vectoriel entre $I_{c u s p}^{s t}(G(n))$ et son analogue stable pour $G L\left(n^{*}, F\right) \times \theta$; cet analogue est défini en [19] V.1 où l'existence de ce facteur direct est démontrée. On sait d'après 19 IV.5 (1) que ce dernier espace s'interprète comme combinaison linéaire de caractères de représentations tempérées $\theta$-stable de $G L\left(n^{*}, F\right)$. Toutefois, 19] ne sait pas que les paramètres de ces représentations sont à valeurs dans $G^{*}(n)$. Pour s'en sortir il faut avoir une description de $I_{c u s p}^{\text {st }}(G)$; cela est fortement suggéré par [2] paragraphe 30 mais n'est pas totalement explicite (cf. notre discussion en 4.3). Cette méthode est certainement la plus conceptuelle mais se heurte à cette difficulté. C'est pour cela que l'on a pris l'autre méthode qui nécessite de devoir montrer que $\tilde{\pi}$ est tempérée ce qui sera fait ci-dessous.

On garde les notations $\psi, \sigma, \psi_{0}$ introduites ci-dessus.

\subsection{Support cuspidal, rappel des définitions et résultats élémentaires}

Soit $\pi$ une série discrète irréductible de $G(n)$. On sait définir le support cuspidal de $\pi$ (comme de toute représentation irréductible de $G(n))$ comme l'union d'une représentation cuspidale irréductible, $\pi_{c u s p}$ d'un groupe $G\left(n_{\text {cusp }}\right)$ (le support cuspidal partiel de $\pi$ ) et d'un ensemble de représentations cuspidales irréductibles $\rho_{i}$ de groupe $G L\left(d_{\rho_{i}}, F\right)$ pour $i$ parcourant un ensemble convenable, $\mathcal{I}$, d'indices. Cet ensemble est défini à permutation près et à inversion près, c'est-à-dire que l'on peut changer $\rho_{i}$ en sa duale. Cet ensemble est uniquement défini par la propriété que $\pi$ est un sous-quotient irréductible de l'induite

$$
\times_{i \in \mathcal{I}} \rho_{i} \times \pi_{\text {cusp }}
$$

On note $\operatorname{Supp}_{G L}(\pi):=\left\{\left(\rho^{\prime \prime}, x\right)\right\}$ l'ensemble des couples $\rho^{\prime \prime}, x$ formés d'une représentation cuspidale unitaire d'un groupe linéaire et d'un réel positif ou nul tel que le support cuspidal de $\pi$ soit l'union de $\pi_{c u s p}$ avec l'ensemble des représentations cuspidales $\rho^{\prime \prime}||^{x} \operatorname{pour}\left(\rho^{\prime \prime}, x\right) \in \operatorname{Supp}_{G L}(\pi)$.

Lemme. (i) Soit $\rho^{\prime \prime}$ une représentation cuspidale unitaire d'un groupe $G L\left(d_{\rho^{\prime \prime}}, F\right)$; on suppose qu'il existe $x^{\prime \prime} \in \mathbb{R}$ tel que $\left(\rho^{\prime \prime}, x^{\prime \prime}\right) \in \operatorname{Supp}_{G L}(\pi)$. Alors, il existe des réels $d, f$ tels que $d-f+1 \in \mathbb{N}_{\geq 1}$ et une série discrète $\pi^{\prime}$ tels que $\pi$ soit un sous-module irréductible de l'induite

$$
<\rho^{\prime \prime}||^{d}, \cdots, \rho^{\prime \prime}||^{f}>\times \pi^{\prime}
$$

où la représentation entre crochet est $S t\left(\rho^{\prime \prime}, d-f+1\right) \|^{(d+f) / 2}$. De plus

$$
\operatorname{Supp}_{G L}(\pi)=\operatorname{Supp}_{G L}\left(\pi^{\prime}\right) \cup\{(\rho,|y|) ; y \in[d, f]\} .
$$

(ii) Pour tout $\left(\rho^{\prime \prime}, x\right) \in \operatorname{Supp}_{G L}(\pi), \rho^{\prime \prime}$ est autoduale et il existe un entier relatif $z$ tel que l'induite $\rho^{\prime \prime}||^{x+z} \times$ $\pi_{\text {cusp }}$ soit réductible.

Ces résultats ne sont pas nouveaux et bien connus des spécialistes. Par définition du support cuspidal pour tout $\left(\rho^{\prime}, x^{\prime}\right) \in \operatorname{Supp}_{G L}(\pi)$, il existe un choix de signe $\zeta_{\left(\rho^{\prime}, x^{\prime}\right)}$ et un ordre sur $\operatorname{Supp} p_{G L}(\pi)$ tel que l'on ait une inclusion :

$$
\pi \hookrightarrow \times_{\left(\rho^{\prime}, x^{\prime}\right) \in \operatorname{Supp}_{G L}(\pi)} \rho^{\prime} \mid \zeta^{\zeta_{\rho^{\prime}, x^{\prime}} x^{\prime}} \times \pi_{\text {cusp }}
$$

On fixe $\rho^{\prime \prime}$ comme dans l'énoncé et on prend pour $f$ le plus petit réel de la forme $\zeta_{\left(\rho^{\prime \prime}, x^{\prime \prime}\right)} x^{\prime \prime}$ tel que $\rho^{\prime \prime}||^{f}$ interviennent dans (1). On peut "pousser" $\left.\rho^{\prime \prime}\right|^{f}$ vers la gauche tout en gardant une inclusion comme dans (1). Et quand il est le plus à gauche possible, toutes les représentations intervenant avant $\rho^{\prime \prime}||^{f}$ sont de la forme $\rho^{\prime \prime}||^{z}$ avec $z$ parcourant un segment de la forme $\left[d, f\left[\right.\right.$ pour $d$ convenable vérifiant $d-f+1 \in \mathbb{N}_{\geq 1}$. Cela entraîne qu'il existe une représentation $\pi^{\prime}$ irréductible et une inclusion de $\pi$ dans l'induite

$$
<\rho^{\prime \prime}||^{d}, \cdots, \rho^{\prime \prime}||^{f}>\times \pi^{\prime} .
$$

Il reste à démontrer que $\pi^{\prime}$ est nécessairement une série discrète. On sait que $d+f>0$ car s'il n'en est pas ainsi, $[d, f]$ est un segment dont le milieu, $(d+f) / 2$ est inférieur ou égal à 0 ; on pose $\delta=0$ si $(d-f+1) / 2$ est un entier et $\delta=(d+f) / 2$ sinon. Ainsi

$$
\sum_{y \in[d, f]} y=\sum_{t \in[1,[(d-f+1) / 2]}(d-t+1+f+t-1)+\delta=[(d-f+1) / 2](d+f)+(d+f) / 2 \leq 0 .
$$


Or le module de Jacquet de $\pi$ contient la représentation $\left.\otimes_{\ell \in[d, f]} \rho\right|^{\ell} \otimes \pi^{\prime}$ et le critère de Casselman montre que les exposants sont une combinaison linéaire à coefficient strictement positif de toutes les racines simples. L'exposant que l'on vient de trouver ne vérifie pas cette positivité d'où notre assertion. Montrons maintenant que $\pi^{\prime}$ est une série discrète; il faut vérifier le critère de Casselman sur les exposants. On suppose a contrario qu'il existe un terme dans le module de Jacquet de $\pi^{\prime}$ de la forme $\otimes \rho^{\prime \prime \prime}||^{x^{\prime \prime \prime}} \otimes \pi_{\text {cusp }}$ qui ne satisfait pas au critère de positivité. Un tel terme donne lieu à une inclusion de $\pi^{\prime}$ dans l'induite $\times \rho^{\prime \prime \prime}||^{x^{\prime \prime \prime}} \times \pi_{\text {cusp }}$. Le critère de positivité de Casselman s'exprime exactement par le fait que pour tout $\rho^{\prime \prime \prime}, x^{\prime \prime \prime}$ intervenant dans cette écriture, $\sum_{(\rho, x)} x>0$ où la somme porte sur les $(\rho, x)$ à gauche de $\left(\rho^{\prime \prime \prime}, x^{\prime \prime \prime}\right)$ y compris $\left(\rho^{\prime \prime \prime}, x^{\prime \prime \prime}\right)$. Supposons que $\pi^{\prime}$ ne soit pas une série discrète. On peut fixer un élément de son module de Jacquet ne vérifiant pas ce critère. Et on peut considérer dans $\sum_{(\rho, x)} x$ comme précédemment, chaque somme partielle où $\rho$ est fixé et l'une au moins est $\leq 0$. Par l'argument déjà donnée on trouve une inclusion de $\pi^{\prime}$ dans une induite de la forme :

$$
<\rho^{\prime}||^{d^{\prime}}, \cdots, \rho^{\prime}||^{f^{\prime}}>\times \pi^{\prime \prime},
$$

où $\left[d^{\prime}, f^{\prime}\right]$ est un segment (i.e. $d^{\prime}-f^{\prime}+1 \in \mathbb{N}_{\geq 1}$ ), $\rho^{\prime}$ est une représentation cuspidale unitaire et $\sum_{x \in\left[d^{\prime}, f^{\prime}\right]} x \leq 0$. Comme ci-dessus, ceci est équivalent à $d^{\prime}+f^{\prime} \leq 0$. L'induite

$$
<\rho^{\prime \prime}||^{d}, \cdots, \rho^{\prime \prime}||^{f}>\times<\rho^{\prime}||^{d^{\prime}}, \cdots, \rho^{\prime}||^{f^{\prime}}>
$$

est nécessairement irrédutible : en effet soit $\rho^{\prime \prime} \nsucceq \rho^{\prime}$ et le résultat est immédiat. Soit $\rho^{\prime \prime}=\rho^{\prime}$, mais alors $f \leq f^{\prime}$ par minimalité de $f$, et

$$
d-d^{\prime}=(d+f)-\left(d^{\prime}+f^{\prime}\right)+\left(f^{\prime}-f\right)>0,
$$

d'où $[d, f] \supset\left[d^{\prime}, f^{\prime}\right]$ et le résultat. On obtient donc une inclusion de $\pi$ dans l'induite :

$$
\begin{gathered}
<\rho^{\prime \prime}||^{d}, \cdots, \rho^{\prime \prime}||^{f}>\times<\rho^{\prime}||^{d^{\prime}}, \cdots, \rho^{\prime}||^{f^{\prime}}>\times \pi^{\prime \prime} \simeq \\
<\rho^{\prime}||^{d^{\prime}}, \cdots, \rho^{\prime}||^{f^{\prime}}>\times<\rho^{\prime \prime}||^{d}, \cdots, \rho^{\prime \prime}||^{f}>\times \pi^{\prime \prime}
\end{gathered}
$$

et $d^{\prime}+f^{\prime} \leq 0$ contredit le fait que $\pi$ est une série discrète. L'assertion sur le $S u p p_{G L}(\pi)$ est claire par définition.

(ii) se déduit de (i) par récurrence : on reprend les notations de (i) et on admet que tous les éléments de $\operatorname{Supp}_{G L}\left(\pi^{\prime}\right)$ sont de la forme $(\rho, x)$ avec $\rho$ autoduale. Si $\rho^{\prime \prime}$ n'est pas autoduale, $\rho^{\prime \prime}||^{x} \times \pi_{\text {cusp }}$ est irréductible pour tout $x$ réel par un résultat général d'Harish-Chandra (cf. [18]) et $\rho^{\prime \prime}||^{x}$ commute avec toute représentation de la forme $\rho^{\prime}||^{ \pm x^{\prime}}$ pour $\left(\rho^{\prime}, x^{\prime}\right) \in \operatorname{Supp}_{G L}\left(\pi^{\prime}\right)$. On aurait donc un isomorphisme :

$$
<\rho^{\prime \prime}||^{d}, \cdots, \rho^{\prime \prime}||^{f}>\times \pi^{\prime} \simeq<\left(\rho^{\prime \prime}\right)^{*-f}, \cdots,\left(\rho^{\prime \prime}\right)^{*-d}>\times \pi^{\prime} .
$$

et une inclusion de $\pi$ dans l'induite de droite. Or $-f-d<0$ ce qui contredit le fait que $\pi$ est une série discrète et le critère de Casselman. C'est exactement le même argument pour la deuxième partie de (ii)

\subsection{Propriétés de demi-integralité du support cuspidal d'une série discrète}

Théorème. (i) Soit $\pi$ une série discrète irréductible de $G(n)$; tout élément $(\rho, x)$ de $\operatorname{Supp}_{G L}(\pi)$ (notation de 1.4) est tel que $\rho$ est autoduale et que $x$ est demi-entier.

(ii) Soit $\pi_{0}$ une représentation cuspidale de $G(n)$ et $\rho$ une représentation cuspidale irréductible autoduale de $G L\left(d_{\rho}, F\right)$ et $x \in \mathbb{R}$ tel que l'induite $\rho||^{x} \times \pi_{0}$ soit réductible. Alors $x \in 1 / 2 \mathbb{Z}$.

Grâce à 1.4 (ii), pour démontrer (i), il suffit de démontrer que pour $\pi_{c u s p}$ une représentation cuspidale irréductible d'un groupe $G\left(n_{\text {cusp }}\right)$ et pour $\rho$ une représentation cuspidale irréductible d'un groupe $G L\left(d_{\rho}, F\right)$, autoduale, le réel positif ou nul, $x_{\rho, \pi_{c u s p}}$ tel que l'induite $\left.\rho\right|^{x_{\rho, \pi_{c u s p}}} \times \pi_{\text {cusp }}$ est réductible, est un demi-entier. C'est-à-dire, en fait, (ii) et c'est donc (ii) que nous allons démontrer.

Soit donc $\pi_{c u s p}$ et $x_{\rho, \pi_{c u s p}}$ comme ci-dessus. Si $x_{\rho, \pi_{c u s p}}=0$, l'assertion est claire. Supposons donc que $x_{\rho, \pi_{\text {cusp }}}>0$. Alors l'induite :

$$
\rho||^{x_{\rho, \pi_{c u s p}}+1} \times \rho||^{x_{\rho, \pi_{c u s p}}} \times \pi_{c u s p}
$$

a un sous-module irréductible qui est une série discrète; c'est un calcul de module de Jacquet facile on coince cette sous-représentation dans l'intersection des 2 sous-représentations suivantes : (la notation $\left\langle\sigma_{1}, \sigma_{2}\right\rangle$ représente le socle de l'induite $\sigma_{1} \times \sigma_{2}$, c'est à dire la somme des sous-modules irréductibles, mais ici le socle est irréductible)

$$
\rho||^{x_{\rho, \pi_{c u s p}}+1} \times<\rho||^{x_{\rho, \pi_{c u s p}}}, \pi_{c u s p}>\cap<\rho||^{x_{\rho, \pi_{c u s p}}+1}, \rho||^{x_{\rho, \pi_{c u s p}}}>\times \pi_{\text {cusp }} .
$$


Puis on calcule les modules de Jacquet, calcul qui prouve d'abord que cette intersection est non nulle puis que c'est une série discrète.

On note $\pi$ cette série discrète et on lui applique 1.3 avec les notations de ce paragraphe. En particulier, si $\psi \neq \psi_{0}, \operatorname{Supp}_{G L}(\pi)$ contient le support cuspidal de $\sigma$ (à "conjugaison" près). Mais $\operatorname{Supp}_{G L}(\pi)$ a exactement 2 éléments $\left.\rho\right|^{x_{\rho, \pi_{c u s p}}+1}$ et $\left.\rho\right|^{x_{\rho, \pi_{c u s p}}}$; cela entraîne déjà que $\sum_{(\rho, a, x) \in \mathcal{I}} a \leq 2$. Si $\mathcal{I}$ est réduit à un élément $(\rho, a, x)$ avec $a=2$, les ensembles $\left(\left(1+x_{\rho, \pi_{\text {cusp }}}\right), x_{\rho, \pi_{\text {cusp }}}\right)$ et $((1 / 2+x),(-1 / 2+x))$ coïncident à l'ordre et au signe près. D'où par positivité, puisque $x<1 / 2$

$$
1+x_{\rho, \pi_{\text {cusp }}}=1 / 2+x ; \quad x_{\rho, \pi_{\text {cusp }}}=1 / 2-x .
$$

Ceci est impossible. On ne pas non plus avoir $|\mathcal{I}|=2$. On sait donc maintenant que soit $\psi=\psi_{0}$ soit $\sigma$ est de la forme $\rho||^{x}$; on rappelle que $\left.x \in\right] 0,1 / 2\left[\right.$ et qu'il cö̈ncide donc avec $x_{\rho, \pi_{\text {cusp }}}$ et non avec $1+x_{\rho, \pi_{\text {cusp }}}$. En d'autres termes soit $\pi \in \Pi(\tilde{\pi})$ avec $\tilde{\pi}=\tilde{\pi}_{0}$ est tempérée soit un sous-quotient irréductible de l'induite $\rho \|^{1+x_{\rho, \pi_{c u s p}} \times \pi_{c u s p}}$ est dans $\Pi\left(\tilde{\pi}_{0}\right)$. En fait l'induite est irréductible par définition de $x_{\rho, \pi_{c u s p}}$ et dans ce dernier cas, ce serait toute l'induite qui serait dans $\Pi\left(\tilde{\pi}_{0}\right)$.

On montre maintenant que tout $\pi_{0} \in \Pi\left(\tilde{\pi}_{0}\right)$ est tel que $\operatorname{Supp}_{G L}\left(\pi_{0}\right)$ est formé de couples $\left(\rho^{\prime}, x^{\prime}\right)$ avec $x^{\prime}$ demi-entier. Cela permettra donc de conclure grâce à ce qui précède; on aura en plus montré que $\sigma$ est trivial.

On fixe $\rho$ une représentation cuspidale unitaire irréductible d'un groupe $G L\left(d_{\rho}, F\right)$. Pour $z \in \mathbb{R}, m \in \mathbb{N}$ et $\pi^{\prime}$ une représentation de $G(m)$ on note $J a c_{z} \pi^{\prime}$ l'unique élément du groupe de Grothendieck des représentations lisses de longueur finie de $G(m)$ tel que la restriction de $\pi^{\prime}$ au Levi $G L\left(d_{\rho}, F\right) \times G(m)$ le long du radical unipotent d'un parabolique de ce Levi soit de la forme $\left.\rho\right|^{z} \otimes J a c_{z} \pi^{\prime} \oplus \tau$ où $\tau$ est une somme de représentations irréductibles de la forme $\rho^{\prime} \otimes \tau^{\prime \prime}$ avec $\rho^{\prime} \not \rho||^{z}$. Pour $\tilde{\pi}$ une représentation de $G L\left(m^{*}+2 d_{\rho^{\prime}}, F\right)$ on définit $J a c_{z}^{\theta} \tilde{\pi}$ comme l'unique élément du groupe de Grothendieck des représentations lisses de longueur finie de $G L(m, F)$ tel que la restriction de $\tilde{\pi}$ au Levi $G L\left(d_{\rho^{\prime}}, F\right) \times G L\left(m^{*}, F\right) \times G L\left(d_{\rho^{\prime}}, F\right)$ soit de la forme $\left.\rho\right|^{z} \otimes J a c_{z}^{\theta} \tau \otimes \rho^{*}||^{-z} \oplus \tau^{\prime}$ où $\tau^{\prime}$ est une somme de représentations irréductibles de la forme $\rho^{\prime} \otimes \tau^{\prime \prime} \otimes \rho^{\prime \prime}$ avec soit $\rho^{\prime} \not \rho||^{z}$ soit $\rho^{\prime \prime} \not \rho^{-z}$. On a vu en [12] (mais c'est assez facile) que l'égalité des traces 1.3 (1) donne aussi une égalité (avec les mêmes coefficients) :

$$
\sum_{\pi \in \Pi\left(\tilde{\pi}_{0}\right)} c_{\pi_{0}} \operatorname{tr} J a c_{z} \pi_{0}(h)=\operatorname{trJac} c_{z}^{\theta} \tilde{\pi}_{0}(g, \theta)
$$

Le terme de droite ne peut être non nul que pour des $z \in 1 / 2 \mathbb{Z}$. Ici on utilise la positivité des $c_{\pi_{0}}$ annoncée par Arthur qui empêche toute simplification dans le terme de gauche. Alors pour $\pi_{0} \in \Pi\left(\tilde{\pi}_{0}\right), J a c_{z} \pi_{0} \neq 0$ nécessite que $J a c_{z}^{\theta} \tilde{\pi}_{0} \neq 0$. D'où le fait que $z \in 1 / 2 \mathbb{Z}$. Si $\operatorname{Supp}_{G L}\left(\pi_{0}\right)$ contient un élément $\left(\rho^{\prime \prime}, x\right)$ avec $x \in \mathbb{R}$,de façon standard (en poussant vers la gauche), on montre qu'il existe $x^{\prime}$ avec $x-x^{\prime} \in \mathbb{Z}$ tel que $J a c_{x^{\prime}} \pi_{0} \neq 0$, en faisant ici $\rho=\rho^{\prime}$. Comme on vient de montrer que $x^{\prime} \in 1 / 2 \mathbb{Z}, x$ aussi est un demi-entier relatif.

Cela termine la preuve du théorème.

\subsection{Morphisme associé à une série discrète}

Le corollaire suivant est évidemment très fortement inspiré par les travaux d'Arthur.

Corollaire. Soit $\pi$ une série discrète irréductible de $G(n)$, alors il existe une représentation tempérée, $\tilde{\pi}$ de $G L\left(n^{*}, F\right), \theta$-discrète telle que $\pi \in \Pi(\tilde{\pi})$. Ou encore, il existe un morphisme $\psi$ de $W_{F} \times S L(2, \mathbb{C})$ dans $G L\left(n^{*}, \mathbb{C}\right)$ tel que $\pi \in \Pi(\psi)$ avec $\psi$ un morphisme $\theta$-discret à valeurs dans $G^{*}(n)$.

En tenant compte de 1.3 il faut montrer que $\tilde{\pi}=\tilde{\pi}_{0}$ avec les notations de ce paragraphe. Comme ci-dessus, nécessairement $\operatorname{Supp}_{G L}(\pi)$ contient le support cuspidal de $\sigma$ (à "conjugaison" près). Mais maintenant on sait que $\operatorname{Supp}_{G L}(\pi)$ est formé de couple $(\rho, x)$ avec $x$ demi-entier et ce n'est pas le cas du support cuspidal de $\sigma$ si $\sigma$ existe vraiment. D'où le fait que $\tilde{\pi}=\tilde{\pi}_{0}$. Le corollaire résulte alors de 1.3 .

\section{Morphismes associés aux représentations cuspidales de $G(n)$ et points de réductibilité des induites de cuspidales.}

On dit qu'un morphisme $\psi$ de $W_{F} \times S L(2, \mathbb{C})$ dans un $G L(m, \mathbb{C})$ est sans trou si pour toute représentation $\rho \otimes \sigma_{a}$ intervenant dans $\psi$ (notations de l'introduction) avec $a>2$ la représentation $\rho \otimes \sigma_{a-2}$ y intervient aussi.

On précise les notations de l'introduction; soit $\pi_{0}$ une représentation cuspidale de $G(m)$ et soit $\rho$ une représentation cuspidale irréductible autoduale de $G L\left(d_{\rho}, F\right)$ ce qui définit $d_{\rho}$. On note $x_{\rho, \pi_{0}}$ l'unique réel positif ou nul (cf. [16]) tel que l'induite $\left.\rho\right|^{x_{\rho, \pi_{0}}} \times \pi_{0}$ soit réductible. Soit $\psi_{0}$ tel que $\pi_{0} \in \Pi\left(\psi_{0}\right)$ ce qui est possible d'après le théorème précédent. Pour $\rho$ comme ci-dessus, on pose

$\operatorname{Jord}_{\rho}\left(\psi_{0}\right):=\left\{a \in \mathbb{N}\right.$; tel que la représentation $\rho \otimes \sigma_{a}$ soit une sous-représentation de $\left.\psi_{0}\right\}$. 
On note $a_{\rho, \psi_{0}}$ l'élément maximal de $\operatorname{Jord}_{\rho}\left(\psi_{0}\right)$ quand cet ensemble est non vide. On dit qu'une représentation de $W_{F} \times S L(2, \mathbb{C})$ (ou simplement de $W_{F}$ ) est du type de $G^{*}$ si elle est orthogonale quand $V$ est symplectique et symplectique quand $V$ est orthogonal.

Corollaire. Soient $\rho, \pi_{0}, \psi_{0}$ comme ci-dessus et tels que $\pi_{0} \in \Pi\left(\psi_{0}\right)$ (cf. 1.9). Alors $\psi_{0}$ est $\theta$ discret et sans trou.

(i) On suppose que $\operatorname{Jord}_{\rho}\left(\psi_{0}\right) \neq \emptyset$ alors $x_{\rho, \pi_{0}}=\left(a_{\rho, \psi_{0}}+1\right) / 2$.

(ii) On suppose que $\operatorname{Jord}_{\rho}\left(\psi_{0}\right)=\emptyset$ mais que $\rho \otimes \sigma_{2}$ est une représentation du type de $G^{*}$; alors $x_{\rho, \pi_{0}}=1 / 2$.

(iii) On suppose que $\operatorname{Jord}_{\rho}\left(\psi_{0}\right)=\emptyset$ et que $\rho$ vue comme représentation de $W_{F}$ est de même type que $G^{*}$. Alors $x_{\rho, \pi_{0}}=0$.

(iv) Jord $\left(\pi_{0}\right)$ (cf introduction pour la notation) coïncide avec Jord $\left(\psi_{0}\right)$. En particulier $\psi_{0}$ est uniquement déterminé par $\pi_{0}$.

Le fait que $\psi_{0}$ est $\theta$ discret est dans 1.3. Le fait que $\psi_{0}$ soit sans trou et les assertions (i) et (ii) sont démontrées en [13. On va redémontrer l'essentielle de ces assertions pour la commodité du lecteur; on ne redonne pas la démonstration de ce que si $\operatorname{Jord}_{\rho}\left(\psi_{0}\right) \neq \emptyset$ alors $x_{\rho, \pi_{0}}=\left(a_{\rho, \psi_{0}}+1\right) / 2$; la démonstration est plus simple mais de même nature que celle de (ii) que l'on va redonner.

En [9], cf. l'introduction, on a montré l'inégalité, où la somme porte sur toutes les représentations cuspidales irréductibles autoduales d'un $G L(d, F)$ :

$$
\sum_{\rho} \sum_{\ell \in\left[1,\left[x_{\rho, \pi}\right]\right]} d_{\rho}\left(2 x_{\rho}-2 \ell+1\right) \leq m^{*} .
$$

Cette somme ne voit que les $\rho$ tel que $x_{\rho}>1 / 2$. Or si on se limite dans le terme de gauche aux représentations $\rho$ telles que $\operatorname{Jord}_{\rho}\left(\psi_{0}\right) \neq \emptyset$, ce terme est supérieur ou égal à $\sum_{(\rho, a) \in \operatorname{Jord}(\psi)} a d_{\rho}$ avec égalité si et seulement si $\operatorname{Jord}\left(\psi_{0}\right)$ est sans trou. Or cette somme vaut $m^{*}$ car c'est exactement la somme des dimensions des sousreprésentations de $\psi_{0}$. Et on a donc démontré que $\psi_{0}$ est sans trou et que pour tout $\rho$ tel que $J_{o r d}\left(\psi_{0}\right)=\emptyset$, $x_{\rho, \pi_{0}} \in[0,1 / 2]$. Comme on sait déjà que $x_{\rho, \pi_{0}}$ est un demi-entier, il reste les possibilités 0 ou $1 / 2$. On remarque ici que le fait que $x_{\rho, \pi_{0}}$ soit entier ou non résulte de la parité de $a_{\rho, \psi_{0}}$ et donc de savoir si le morphisme de $W_{F}$ dans $G L\left(d_{\rho}, \mathbb{C}\right)$ associé à $\rho$ est du type de $G^{*}$ ou non. Ce sont les conditions de parités voulues dans la définition des blocs de Jordan.

Avec cela (iv) résulte de [9] 4.3.

Il reste donc à montrer, pour toute représentation autoduale cuspidale irréductible, $\rho$, telle que $\operatorname{Jord}_{\rho}\left(\psi_{0}\right)=$ $\emptyset$, l'équivalence entre le fait que $\rho$ est telle que $x_{\rho, \pi_{0}}=1 / 2$ et le fait que $\rho \otimes \sigma_{2}$ est de même type que $G^{*}$. On rappelle que $\sigma_{2}$ est une représentation symplectique et donc que $\rho \otimes \sigma_{2}$ est une représentation symplectique si $\rho$ est une représentation orthogonale et est une représentation orthogonale si $\rho$ est une représentation symplectique.

Soit donc $\rho$ tel que $x_{\rho, \pi_{0}}=1 / 2$; on note ici $\pi_{d}$ l'unique sous-module irréductible de l'induite $\rho \|^{1 / 2} \times \pi_{0}$; c'est une série discrète par hypothèse de réductibilité. On considère une représentation tempérée, $\theta$-discrète, $\tilde{\pi}_{d}$ telle que $\pi \in \Pi\left(\tilde{\pi}_{d}\right)$ et on écrit avec des coefficients $c_{\pi^{\prime}}$ positifs, comme en 1.3

$$
\sum_{\pi^{\prime} \in \Pi\left(\tilde{\pi}_{d}\right)} c_{\pi^{\prime}} \operatorname{tr} \pi^{\prime}(h)=\operatorname{tr} \tilde{\pi}_{d}(g, \theta) .
$$

Comme $\tilde{\pi}_{d}$ est une représentation tempérée, on l'écrit comme une induite de représentations de Steinberg généralisées, $\times{ }_{\left(\rho^{\prime}, a\right) \in \mathcal{I}} S t\left(\rho^{\prime}, a\right)$ où $\mathcal{I}$ est un ensemble d'indices paramétrant des couples $\left(\rho^{\prime}, a\right)$ formés d'une représentation cuspidale unitaire $\rho^{\prime}$ et d'un entier $a$; on sait que $\mathcal{I}$ est sans multiplicité puisque $\tilde{\pi}_{d}$ est $\theta$-discret. Avec les notations $J a c_{z}$ et $J a c_{z}^{\theta}$ de la preuve de 1.6 pour $\rho$ fixé comme ici, on a encore que $J a c_{1 / 2}^{\theta} \tilde{\pi}_{d}$ est un transfert de

$$
\sum_{\pi^{\prime} \in \Pi(\tilde{\pi})} c_{\pi^{\prime}} \operatorname{tr} J a c_{1 / 2} \pi^{\prime} .
$$

Or $J a c_{1 / 2} \pi_{d}=\pi_{0}$, ainsi $J a c_{1 / 2}^{\theta} \tilde{\pi}_{d} \neq 0$. Cela nécessite que $\mathcal{I}$ contienne $(\rho, 2)$; il le contient avec multiplicité exactement 1 . On obtient alors :

$$
J a c_{1 / 2}^{\theta} \tilde{\pi}_{d}=\times_{\left(\rho^{\prime}, a\right) \in \mathcal{I}-\{(\rho, 2)\}} S t\left(\rho^{\prime}, a\right)=: \tilde{\pi}_{0}
$$

et $\pi_{0} \in \Pi\left(\tilde{\pi}_{0}\right)$. On note encore $\psi_{0}$ le morphisme associé à $\pi_{0}$ et le paramètre de $\tilde{\pi}_{d}$ est donc la somme de $\psi_{0}$ et de $\rho \otimes \sigma_{2}$ (ici $\rho$ est la représentation irréductible de $W_{F}$ associée à $\rho$ via la correspondance de Langlands et $\sigma_{2}$ est la représentation irréductible de dimension 2 de $S L(2, \mathbb{C})$ ). D'après la description d'Arthur (formule (30.15) de 30.2) ce paquet doit être de même type que $G^{*}$; comme c'est déjà le cas de $\psi_{0}$, il en est de même de $\rho \otimes \sigma_{2}$. 
On suppose maintenant que $\rho$ est tel que $\rho \otimes \sigma_{2}$ est de même type que $G^{*}$ et que $\operatorname{Jord}_{\rho}(\psi)=\emptyset$; on doit montrer que $x_{\rho, \pi_{0}}=1 / 2$. On note $\psi_{d}:=\rho \otimes \sigma_{2} \oplus \psi_{0}$ le morphisme de $W_{F} \times S L(2, \mathbb{C})$ dans $G L\left(m^{*}+2 d_{\rho}, \mathbb{C}\right)$ et $\tilde{\pi}_{d}$ la représentation tempérée de $G L\left(m^{*}+2 d_{\rho}, F\right)$ lui correspondant. Le morphisme $\psi_{d}$ est à valeurs dans $G^{*}\left(m+d_{\rho}\right)$. On a donc encore un transfert, pour des bons coefficients positifs ([2] 30.1)

$$
\sum_{\pi^{\prime} \in \Pi\left(\psi_{d}\right)} c_{\pi^{\prime}} \operatorname{tr} \pi^{\prime}(h)=\operatorname{tr} \tilde{\pi}_{d}(g, \theta) .
$$

Et avec les notations $J a c_{z}$ et $J a c_{z}^{\theta}$ déjà introduite pour $\rho$ fixé et $z \in \mathbb{R}$ :

$$
\sum_{\pi^{\prime} \in \Pi\left(\psi_{d}\right)} c_{\pi^{\prime}} J a c_{z} \operatorname{tr} \pi^{\prime}\left(h^{\prime}\right)=J a c_{z}^{\theta} \operatorname{tr} \tilde{\pi}_{d}\left(g^{\prime}, \theta\right) .
$$

On l'applique d'abord avec $z=1 / 2$; le terme de droite n'est pas nul et il contient $\tilde{\pi}_{0}$; il est même réduit à $\tilde{\pi}_{0}$ car par hypothèse $\operatorname{Jord}_{\rho}\left(\pi_{0}\right)=0$ et on a déjà identifié $\operatorname{Jord}_{\rho}\left(\pi_{0}\right)$ avec les entiers $a$ tel que $\rho \otimes \sigma_{a}$ soit une sous-représentation de $\psi_{0}$. Donc $\psi_{0}$ ne contient pas $\rho \otimes \sigma_{2}$. Ainsi le terme de gauche de (2) est le paquet associé à $\psi_{0}$ et il contient donc $\pi_{0}$. Ainsi il existe $\pi^{\prime} \in \Pi\left(\psi_{d}\right)$ tel que $J a c_{1 / 2} \pi^{\prime}$ contienne $\pi_{0}$ ou encore que $\pi^{\prime}$ est un sous-quotient irréductible de $\left.\rho\right|^{1 / 2} \times \pi_{0}$. Comme $J a c_{1 / 2} \pi^{\prime} \neq 0, \pi^{\prime}$ est un sous-module irréductible de l'induite $\rho||^{1 / 2} \times \pi_{0}$. Cette induite est nécessairement réductible car sinon $J a c_{-1 / 2} \pi^{\prime} \neq 0$ alors que pour $z=-1 / 2$ le terme de droite de (2) est nul. Ainsi $x_{\rho, \pi_{0}}=1 / 2$ comme cherché et cela termine la démonstration.

\section{Classification et paquet de Langlands}

Soit $\pi$ une série discrète irréductible de $G(n)$; nous lui avons associé un morphisme de $W_{F} \times S L(2, \mathbb{C})$ dans $G L\left(n^{*}, \mathbb{C}\right)$, à l'aide de ses blocs de Jordan. On note $\psi_{\pi}$ ce morphisme. En suivant Arthur, on lui a aussi associé un morphisme en 1.6, le théorème ci-dessous dit que ces morphismes sont conjugués :

Théorème. On a $\pi \in \Pi\left(\psi_{\pi}\right)$.

Ce théorème montre que nos constructions sont compatibles avec celles d'Arthur et que le morphisme associé à une série discrète à l'aide de ses blocs de Jordan est bien celui conjecturé par Langlands. Toutefois, il n'est pas démontré que les coefficients permettant de construire la distribution stable associée au paquet sont égaux à 1 ; ce problème est résolu par Waldspurger en [20] pour certaines représentations venant des constructions de Lusztig $([\S])$ et pour $G(n)=S O(2 n+1, F)$. Et il n'est pas non plus démontré que les représentations à l'intérieur du paquet sont classifiées par les caractères du centralisateur; ce point semble moins sérieux et nous règlerons le cas des groupes orthogonaux déployés ici avec une méthode tout à fait générale.

Pour éviter les confusions, on note $\psi$ plutôt que $\psi_{\pi}$ le morphisme associé à $\pi$ par [10] et [11] à l'aide des blocs de Jordan; on rappellera les propriétés qui le caractérise ci-dessous. Et on note $\tilde{\psi}$ celui associé essentiellement par Arthur en 1.6 et $\tilde{\pi}$ la représentation tempérée de $G L\left(n^{*}, F\right)$ associé à $\tilde{\psi}$. Il faut démontrer que $\psi$ et $\tilde{\psi}$ sont conjugués. On réutilise les notations $J a c_{z}$ et $J a c_{z}^{\theta}$ de 1.5. On vérifie d'abord que $\tilde{\psi}$ est $\theta$-discret. Sinon $\pi$ serait un sous-quotient d'une induite de la forme $S t(\rho, a) \times \pi^{\prime}$ avec $\pi^{\prime}$ une représentation au moins unitaire; l'induite est alors semi-simple et $\pi$ est tempéré mais non discret. Ainsi pour tout $z \in \mathbb{R}, J a c_{z}^{\theta} \tilde{\pi}$ est 0 ou est une représentation tempérée irréductible. On a vu en loc. cit. que pour tout $z \in \mathbb{R}$,

$$
J a c_{z} \pi \in \Pi\left(J a c_{z}^{\theta} \tilde{\pi}\right) .
$$

Si $\pi$ est une représentation cuspidale, le fait que $\psi$ et $\tilde{\psi}$ sont conjugués a été vu en 2 (iv). On suppose donc que $\pi$ n'est pas cuspidale. D'après la construction de [10], 2 cas sont à distinguer. Dans ce qui suit $\rho$ est une représentation cuspidale autoduale irréductible d'un $G L\left(d_{\rho}, F\right)$.

1e cas : il existe $x_{0}$ de la forme $(a-1) / 2$ avec $a \in \mathbb{N}_{>1}$ et une série discrète $\pi^{\prime}$ tel que $\pi$ soit l'unique sous-module irréductible de l'induite $\rho||^{x_{0}} \times \pi^{\prime}$. Dans ce cas, si on note $\psi^{\prime}$ le morphisme de $W_{F} \times S L(2, \mathbb{C})$ dans $G^{n^{*}-d_{\rho}}$, alors $\psi^{\prime}$ contient $(\rho, a-2)$ comme bloc de Jordan ( $a=2$ est accepté) et $\psi$ s'obtient en remplaçant ce bloc de Jordan par $(\rho, a)$.

Concluons dans ce cas ; comme $J a c_{x=(a-1) / 2}^{\theta} \tilde{\pi}_{v} \neq 0$ et est irréductible, cela veut dire que la représentation $\rho \otimes \sigma_{a}$ intervient avec multiplicité exactement 1 dans $\tilde{\psi}$ et que la représentation obtenue s'obtient simplement en remplaçant $\tilde{\psi}$ par un morphisme $\tilde{\psi}^{\prime}$ ayant les mêmes sous-représentations irréductibles sauf cette représentation qui devient $\rho \otimes \sigma_{a-2}$. Il suffit d'appliquer par exemple une hypothèse de récurrence pour savoir que $\tilde{\psi}^{\prime}$ et $\psi^{\prime}$ sont conjugués pour obtenir la même assertion pour $\tilde{\psi}$ et $\psi$. 
2e cas : il existe $x_{0}$ de la forme $(a+1) / 2$ avec $a \in \mathbb{N}_{\geq 1}$ et une série discrète $\pi^{\prime}$ tel que $\pi$ soit l'un des 2 sous-modules irréductibles de l'induite :

$$
<\rho \|^{(a+1) / 2}, S t(\rho, a)>\times \pi^{\prime}
$$

On note $\psi^{\prime}$ le morphisme associé à $\pi^{\prime}$ par nos construction et on admet encore par récurrence que $\pi^{\prime} \in \Pi\left(\psi^{\prime}\right)$. La différence avec le cas 1 est que $J a c_{x_{0}} \pi$ est une représentation $\pi_{1}$ qui est une représentation tempérée et non plus une série discrète. Toutefois, nous avons déjà vérifié que puisque $\pi^{\prime} \in \Pi\left(\psi^{\prime}\right)$, alors $\pi_{1} \in \Pi\left(\psi^{\prime} \oplus\left(\rho \otimes \sigma_{a} \oplus \rho \otimes \sigma_{a}\right)\right)$ et comme ci-dessus,

$$
\tilde{\psi}=\psi^{\prime} \oplus \rho \otimes \sigma_{a+2} \oplus \rho \otimes \sigma_{a} .
$$

Mais d'après nos constructions, on a bien que $\operatorname{Jord}(\psi)$ se déduit de $\operatorname{Jord}\left(\psi^{\prime}\right)$ en ajoutant les 2 blocs $(\rho, a)$ et $(\rho, a+2)$ ce qui est exactement la même chose. Ceci prouve le théorème.

\section{Classification à la Langlands des séries discrètes de $S O(2 n+1, F)$.}

Dans cette partie on suppose que $G(n)$ est la forme déployée du groupe $S O(2 n+1, F)$. On a en vue la classification de Langlands des séries discrètes. Soit $\psi$ un morphisme $\theta$-discret de $W_{F} \times S L(2, \mathbb{C})$ dans $G L(2 n, \mathbb{C})$, on dit qu'il est $\theta$-stable s'il se factorise par $S p(2 n, \mathbb{C})$.

\subsection{Définition de l'espace $I_{c u s p}()$}

On note $C_{\text {cusp }}(G)$ l'ensemble des fonctions lisses cuspidales de $G$, c'est-à-dire celles dont les intégrales orbitales sur les éléments non elliptiques sont nulles et $I_{c u s p}(G)$ est l'image de cet espace vectoriel de fonctions modulo le sous-espace des fonctions lisses dont toutes les intégrales orbitales sont nulles. L'article d'Arthur [3] montre toute l'importance de cet espace; dans cet article le groupe est supposé connexe, ce qui est le cas de $G$. On définit $I_{c u s p}^{\text {st }}(G)$ comme l'ensemble des éléments qui ont des intégrales orbitales constantes sur les classes de conjugaison stable. On appelle $I_{c u s p}^{n s}(G)$ l'ensemble des éléments dont la somme des intégrales orbitales sur toute classe de conjugaison stable est nulle. Waldspurger a remarqué (cf. [12] 4.5) que sans aucun lemme fondamental, on peut montrer la décomposition :

$$
I_{\text {cusp }}(G)=I_{c u s p}^{s t}(G) \oplus I_{c u s p}^{n s}(G) .
$$

Le point est que la stabilité commute à la transformation de Fourier [17]. Pour aller plus loin, on suppose la validité des lemmes fondamentaux pour les groupes endoscopiques de $G$ les lemmes fondamentaux et pour les algèbres de Lie, introduits par Waldspurger. Pour toute donnée endoscopique, noté abusivement $H$ de $G$, on peut d'une part définir $I_{c u s p}^{\text {st }}(H)$ (en remplaçant $G$ par $H$ dans les notations ci-dessus) et un transfert de $I_{c u s p}^{\text {st }}(H)$ dans $I_{c u s p}(G)$; on note $I_{c u s p}^{H-s t}(G)$ l'image; cette image peut se définir directement. Et le résultat principal de [3] est de prouver l'égalité :

$$
I_{\text {cusp }}^{n s}(G)=\oplus_{H} I_{\text {cusp }}^{H-s t}(G) .
$$

Waldspurger définit et étudie l'espace analogue à $I_{c u s p}(G)$ pour les groupes non connexes de la forme $\tilde{G L}\left(m^{\prime}, F\right)$ et montre en [19] VI.1 que sous l'hypothèse d'un lemme fondamental convenable, le transfert induit un isomorphisme de $I_{\text {cusp }}^{\text {st }}(G)$ sur $I_{\text {cusp }}^{\text {st }}(\tilde{G L}(2 n, F))$. On peut ainsi récrire (1) et $(2)$ en

$$
I_{\text {cusp }}(G)=\oplus_{H} I_{\text {cusp }}^{H-s t}(G),
$$

où ici $H$ parcourt toutes les données endoscopiques en incluant pour la partie stable $H=\tilde{G L}(2 n, F)$.

\subsection{Représentations elliptiques et $I_{c u s p}$}

Soit $\pi$ une série discrète; on sait qu'elle possède un pseudo-coefficient et on peut définir la projection de ce pseudo coefficient sur l'ensemble des fonctions cuspidales; pour le cas le plus nouveau celui de $\tilde{G L}(2 m, F)$ ceci est expliqué en [19] II. Ceci s'étend aux représentations elliptiques et permet de définir pour toute représentation elliptique un élément de $I_{c u s p}(G) ; I_{c u s p}(G)$ est engendré comme espace vectoriel par ces images.

Soit maintenant $\psi$ un morphisme de $W_{F} \times S L(2, \mathbb{C})$ dans $S p(2 n, \mathbb{C})$; on supppose que $\psi$ est $\theta$-discret, c'està-dire que la représentation définie quand on inclut $\operatorname{Sp}(2 n, \mathbb{C})$ dans $G L(2 n, \mathbb{C})$ est sans multiplicité; pour aller plus vite on appelle un tel morphisme $\theta$-stable (car il est à image dans $S p(2 n, \mathbb{C})$ ) et $\theta$-discret. On a alors vérifié que les éléments de $\Pi(\psi)$ sont des séries discrètes.

On suppose maintenant que $\psi$ n'est pas $\theta$-discret; $\Pi(\psi)$ est toujours défini uniquement par la propriété de transfert stable. Puisque $\psi$ n'est pas $\theta$-discret, $\psi$ est à valeurs dans un sous-groupe de Levi d'un parabolique 
$\theta$-stable et les éléments de $\Pi(\psi)$ sont les sous-modules irréductibles d'une induite de représentations tempérées; en particulier $\Pi(\psi)$ ne contient pas de séries discrètes.

En général, on note $I_{c u s p}(G)[\psi]$ l'espace vectoriel engendré par l'image dans $I_{c u s p}(G)$ des représentations elliptiques combinaisons linéaires de représentations incluses dans $\Pi(\psi)$; cet espace peut-être 0 . Mais si $\psi$ est $\theta$-discret on a :

$$
\operatorname{dim} I_{\text {cusp }}(G)[\psi]=|\Pi(\psi)| \text {. }
$$

On a vérifié en 3 que pour $\pi$ une série discrète, un morphisme $\psi$ tel que $\pi \in \Pi(\psi)$ est uniquement déterminé par les blocs de Jordan de $\pi$; en particulier si $\Pi(\psi)$ contient une série discrète alors pour tout morphisme $\psi^{\prime}$ $\theta$-stable, $\Pi(\psi) \cap \Pi\left(\psi^{\prime}\right)=\emptyset$.

On pose $I_{c u s p}(G)[n d]$ la somme des espaces $I_{c u s p}(G)[\psi]$ pour tous les morphismes $\psi$ non $\theta$-discret. On a donc la décomposition en somme directe :

$$
I_{\text {cusp }}(G)=\oplus_{\psi} I_{\text {cusp }}(G)[\psi] \oplus I_{\text {cusp }}(G)[n d] .
$$

\subsection{Description de $I_{\text {cusp }}^{\text {st }}(G)$}

Pour tout morphisme $\psi \theta$-stable et $\theta$-discret, en [2] 30.1 (30.14) (pour $s=1$ ), il est construit un élément de $I_{\text {cusp }}^{\text {st }}(G)[\psi]$. On a besoin d'avoir que $I_{c u s p}^{\text {st }}(G)$ est engendré par ces éléments. Ceci n'est pas écrit dans [2] mais est très fortement suggéré par les résultats de loc.cit. pour les raisons suivantes.

Pour tout morphisme $\psi, \theta$-discret mais non nécessairement $\theta$-stable, on note $I_{\text {cusp }}(\tilde{G})[\psi]$ le sous-espace vectoriel de dimension 1 de $I_{c u s p}(\tilde{G L}(2 n, F))$ engendré par l'image d'un prolongement à $\tilde{G L}(2 n, F)$ de la représentation tempérée de $G L(2 n, F)$ définie par $\psi$. Waldspurger a montré que $I_{c u s p}(G L(2 n, F))$ est engendré par ces sous-espaces vectoriels. Le problème est donc de savoir si $I_{c u s p}^{s t}(\tilde{G L}(2 n, F))$ est engendré par ceux de ces éléments qui correspondent aux $\psi, \theta$-stable. A $\psi$ simplement $\theta$-discret, Arthur lui-même associe un groupe endoscopique elliptique de $\tilde{G L}(2 m, F)$ en [2] pages 235 et 236 : on décompose $\psi$ en la somme de 2 morphismes $\psi_{s} \oplus \psi_{o}$ où $\psi_{s}$ est à valeurs dans un groupe symplectique, $\operatorname{Sp}\left(2 m_{s}, \mathbb{C}\right)$ alors que $\psi_{o}$ est à valeurs dans un groupe orthogonal, $O\left(2 m_{o}, \mathbb{C}\right)$. Le déterminant de la restriction de $\psi_{o}$ à $W_{F}$ donne un caractère quadratique $\eta$ du corps de base (par réciprocité) et le groupe endoscopique est déterminé par $m_{s}, m_{o}$ et $\eta$. On note $H_{\psi}$ ce groupe endoscopique, c'est le groupe $S p\left(2 m_{s}, F\right) \times O\left(2 m_{o}, F\right)$ où le groupe orthogonal est le groupe de la forme orthogonale de dimension $2 m_{o}$ de discriminant (normalisé) $\eta$ et d'invariant de Hasse est +1 . Par 30.1, Arthur associe à $\psi$ un élément stable de $I_{\text {cusp }}\left(H_{\psi}\right)$ puisque l'on est dans une situation produit mais il ne transfert pas cet élément en un élément de $I_{c u s p}^{H_{\psi}-s t}(\tilde{G L}(2 m, F))$. Comme on admet les lemmes fondamentaux on peut bien faire ce transfert et le point est de montrer que ce transfert coïncide avec l'image de $\pi(\psi) \circ \theta$ dans $I_{c u s p}\left(\tilde{G}_{m}\right)$. On admet donc pour la suite de cette partie l'hypothèse suivante :

$I_{\text {cusp }}^{\text {st }}(\tilde{G L}(2 n, F))$ est la somme des espaces $I_{\text {cusp }}(\tilde{G})[\psi]$, où $\psi$ parcourt l'ensemble des morphismes $\theta$-stables et $\theta$-discrets.

La conséquence de cette hypothèse est que la décomposition 4.2 (2) est compatible à la projection sur $I_{\text {cusp }}^{\text {st }}(G)$ et précisément que l'on a :

$$
I_{\text {cusp }}^{\text {st }}(G)=\oplus_{\psi}\left(I_{\text {cusp }}(G)[\psi] \cap I_{\text {cusp }}^{\text {st }}(G)\right)
$$

où $\psi$ parcourt l'ensemble des classes de conjugaison de morphismes $\theta$-stables et $\theta$-discrets.

Montrons que cette hypothèse entraîne aussi la décomposition en somme directe pour tout $\psi$ morphisme $\theta$-stable et $\theta$-discret :

$$
I_{\text {cusp }}(G)[\psi]=\oplus_{H}\left(I_{\text {cusp }}^{H-s t}(G) \cap I_{\text {cusp }}(G)[\psi]\right),
$$

où $H$ parcourt le même ensemble qu'en 4.1 (3).

Ceci n'étant pas directement dans 2 30.1, il faut le vérifier. Et il suffit de prouver l'analogue de (1) pour $H$ un groupe endoscopique de $S p(2 m, F)$; pour un tel groupe il faut aussi tenir compte de $I_{c u s p}(G)[n d]$. Soit $H$ un produit de groupes orthogonaux de la forme $S O\left(2 m_{1}+1, F\right) \times S O\left(2 m_{2}+1, F\right)$ avec $m=m_{1}+m_{2}$; on applique la propriété (1) à chaque facteur pour décrire $I_{c u s p}^{s t}(H)$ comme somme directe des espaces vectoriels de dimension $1, I_{\text {cusp }}^{\text {st }}(H)\left[\psi_{1} \times \psi_{2}\right]$, où $\psi_{i}$, pour $i=1,2$ est un morphisme $\theta$-stable et $\theta$-discret à valeurs dans $S p\left(2 m_{i}, \mathbb{C}\right)$. On sait transférer un tel espace vectoriel dans $I_{c u s p}(G)$ grâce à [2] 30.1 (30.14); le transfert est à valeurs dans 
$I_{\text {cusp }}(G)\left(\psi_{1,2}\right)$ où $\psi_{1,2}$ est le morphisme de $W_{F} \times S L(2, \mathbb{C})$ dans $S p(2 m, \mathbb{C})$ obtenu en composant $\psi_{1} \times \psi_{2}$ avec l'inclusion naturelle de $S p\left(2 m_{1}, \mathbb{C}\right) \times S p\left(2 m_{2}, \mathbb{C}\right)$ dans $S p(2 m, \mathbb{C})$. Cela donne exactement la décomposition :

$$
I_{c u s p}^{H-s t}(G)=\oplus_{\psi}\left(I_{c u s p}(G)[\psi] \cap I_{c u s p}^{H-s t}(G)\right) \oplus\left(I_{c u s p}(G)[n d] \cap I_{c u s p}^{H-s t}(G)\right) .
$$

On en déduit la double somme :

$$
I_{\text {cusp }}(G)=\oplus_{H}\left(\oplus_{\psi}\left(I_{\text {cusp }}(G)[\psi] \cap I_{\text {cusp }}^{H-s t}(G)\right) \oplus I_{\text {cusp }}(G)[n d] \cap I_{\text {cusp }}^{H-s t}(G)\right) .
$$

Et on peut inverser les sommations pour obtenir le résultat cherché (2).

\subsection{Classification des séries discrètes}

On rappelle que l'on a admis tous les lemmes fondamentaux de notre situation et l'hypothèse de 4.3 .

Théorème. Soit $\psi$ un morphisme $\theta$-stable et $\theta$-discret que l'on voit comme une représentation de $W_{F} \times$ $S L(2, \mathbb{C})$ à valeurs dans $G L(2 m, \mathbb{C})$. On note $\ell_{\psi}$ le nombre de sous-représentations irréductibles incluses dans $\psi$. Alors :

$$
|\Pi(\psi)|=2^{\ell_{\psi}-1} .
$$

Et l'application définie par Arthur qui associe un caractère du centralisateur de $\psi$ dans $S p(2 n, \mathbb{C})$ est une bijection sur l'ensemble des caractères de restriction triviale au centre de $\operatorname{Sp}(2 n, \mathbb{C})$.

On sait déjà que $I_{\text {cusp }}^{\text {st }}(G)[\psi]$ est de dimension 1 . On fixe $H=S O\left(2 m_{1}+1, F\right) \times S O\left(2 m_{2}+1, F\right)$ et on doit calculer le nombre de couples $\psi_{1}, \psi_{2}$ tels que $\psi_{i}$ est $\theta$-stable et $\theta$-discret pour le groupe $S O\left(2 m_{i}+1, F\right)$ (pour $i=1,2)$ et tels $\psi$ soit conjugué de $\psi_{1} \times \psi_{2}$ puisque chacun de ces couples donnent un élément de $I_{c u s p}^{H-s t}(G)[\psi]$ et que cela l'engendre complètement. Il est plus simple de faire ce calcul en laissant varier la décomposition de $m$ en $m_{1}+m_{2}$; ensuite il faut diviser le résultat par 2 à cause des isomorphismes entre groupes endoscopiques. Ici on autorise $m_{1} m_{2}=0$, c'est-à-dire que l'on retrouve 2 fois la partie correspondant à $I_{\text {cusp }}^{\text {st }}(G)[\psi]$. On note $\operatorname{Jord}(\psi)$ l'ensemble des sous-représentations irréductibles de $W_{F} \times S L(2, \mathbb{C})$ incluses dans $\psi$; on rappelle que c'est un ensemble sans multiplicité. Le nombre de décomposition de $\psi$ en $\psi_{1} \times \psi_{2}$ est précisément le nombre de décomposition de $\operatorname{Jord}(\psi)$ en 2 sous-ensembles, c'est à dire $2^{\ell_{\psi}}$ puisque $\ell_{\psi}$ est le nombre d'éléments de $J$ ord $(\psi)$ par définition. On obtient le nombre d'élément de $\Pi(\psi)$ quand on a divisé par 2.

On vérifie aisément que le cardinal du centralisateur de $\psi$ dans $S p(2 m, \mathbb{C})$ est $2^{\ell_{\psi}}$ et que le centre est de cardinal 2. Ainsi le groupe des caractères du centralisateur de $\psi$ dans $S p(2 m, \mathbb{C})$ triviaux sur le centre de $S p(2 m, \mathbb{C})$ est aussi de cardinal $2^{\ell_{\psi}-1}$. Pour démontrer la deuxième assertion du théorème, il suffit donc de montrer que l'application définie par Arthur est surjective. On note $\epsilon_{\mathcal{A}}(\pi)$ cette application. Ce que l'on connaît est le rang de la matrice dont les lignes sont indéxées par les éléments de $\operatorname{Centr}_{S p(2 m, \mathbb{C})} \psi / \operatorname{Cent}(\operatorname{Sp}(2 m, \mathbb{C}))$ et les colonnes par les éléments de $\Pi(\psi)$, les coefficients de la matrice étant

$$
\epsilon_{\mathcal{A}}(\pi)(s) ; s \in \operatorname{Centr}_{S p(2 m, \mathbb{C})} \psi / \operatorname{Cent}(\operatorname{Sp}(2 m, \mathbb{C})), \pi \in \Pi(\psi) .
$$

C'est une matrice carré. Vérifions que le rang de cette matrice est le nombre de ses lignes : fixons une ligne donc $s \in \operatorname{Centr}_{S p(2 m, \mathbb{C})} \psi / \operatorname{Cent}(\operatorname{Sp}(2 m, \mathbb{C}))$. Un tel élément fixe une décomposition de $\psi$ en $\psi_{1} \times \psi_{2}$, où pour $i=1,2, \psi_{i}$ est inclus dans l'espace propre pour l'une des 2 valeurs propres de $s$; comme $s$ n'est défini que modulo le centre de $S p(2 m, \mathbb{C})$, cette décomposition n'est définie qu'à l'échange près des 2 facteurs. L'élément $s$ définit aussi un groupe endoscopique, $H_{s}$, à isomorphisme près (qui est le groupe $\tilde{G L}(2 m, F)$ si $s$ est central). Et l'élément

$$
\sum_{\pi} \epsilon_{\mathcal{A}}(\pi)(s) \pi
$$

a pour image dans $I_{c u s p}(G)$ l'élément [2] (30.14) correspondant à $H_{s}$ et à la décomposition $\psi=\psi_{1} \times \psi_{2}$ de $\psi$. Réciproquement à toute décomposition de $\psi$ en $\psi_{1} \times \psi_{2}$, on associe un élément $s$ simplement en donnant ses 2 espaces propres comme ci-dessus. Ainsi les éléments $(*)_{s}$ donne la base de $I_{c u s p}(G)[\psi]$ déjà considérée. Ils sont linéairement indépendants et forment un ensemble de cardinal $2^{\ell_{\psi}-1}$ puisque ce nombre est la dimension de l'espace vectoriel $I_{c u s p}(G)[\psi]$. Cela prouve notre assertion sur le rang de la matrice et termine la preuve. 


\subsection{Caractère et module de Jacquet}

On fixe $\psi$ un morphisme $\theta$-stable et $\theta$-discret. On reprend les notations de l'introduction; soit $\rho \otimes \sigma_{a}$ une sous-représentation irréductible de $\psi$ vu comme représentation de $W_{F} \times S L(2, \mathbb{C})$. Dans l'introduction on a défini l'entier $a_{-}$dans les 2 cas suivants :

soit il existe $b \in \mathbb{N}$ tel que $\rho \otimes \sigma_{b}$ soit une sous-représentation irréductible de $\psi$ avec $b<a_{-}$, auquel cas on pose $a_{-}$le plus grand des entiers $b$ avec ces propriétés

soit il n'existe pas d'entier $b$ comme ci-dessus mais $a$ est pair et on pose $a_{-}=0$.

On a noté $Z_{\rho \otimes \sigma_{a}}$ le sous-groupe à 2 éléments du centralisateur de $\psi$ dans $S p(2 n, \mathbb{C})$ qui correspond à cette sous-représentation $\rho \otimes \sigma_{a}$; précisément cette représentation est à valeurs dans un sous-groupe symplectique de $S p(2 n, \mathbb{C})$ et $Z_{\rho \otimes \sigma_{a}}$ est le centre de ce sous-groupe.

Soit $\pi \in \Pi(\psi)$ et notons $\epsilon_{\mathcal{A}}(\pi)$ le caractère du centralisateur de $\psi$ qu'Arthur associe à $\pi ; \epsilon_{\mathcal{A}}(\pi)$ est connu quand on connaît toutes ses restrictions aux sous-groupes $Z_{\rho \otimes \sigma_{a}}$. On avait remarqué en 10 que ce caractère devait être lié aux propriétés de modules de Jacquet de $\pi$. C'était le point de départ des classifications de [10 et [11] et on va montrer que $\epsilon_{\mathcal{A}}(\pi)$ a bien la propriété qui permet de définir les caractères en loc. cit. ou encore que $\epsilon_{\mathcal{A}}(\pi)$ coïncide avec le caractère associé à $\pi$ par 110 là où nous l'avions défini.

Pour unifier le théorème ci-dessous, on dit, par convention que la restriction d'un caractère à $Z_{\rho \otimes \sigma_{a}}$ est le caractère trivial si $a_{-}=0$, cas où $Z_{\rho \otimes \sigma_{a_{-}}}$n'est pas le groupe $\{ \pm 1\}$. On note $d_{\rho}$ la dimension de la représentation $\rho$ et on note encore $\rho$ la représentation cuspidale de $G L\left(d_{\rho}, F\right)$ associée à $\rho$ par la correspondance de Langlands.

Théorème. Soit $\rho \otimes \sigma_{a}$ une sous-représentation de $\psi$ telle que $a_{-}$soit défini. Alors, la restriction de $\epsilon_{\mathcal{A}}(\pi)$ à $Z_{\rho \otimes \sigma_{a}} \simeq\{ \pm 1\}$ est le même caractère que la restriction de $\epsilon_{\mathcal{A}}(\pi)$ à $Z_{\rho \otimes \sigma_{a_{-}}}$si et seulement si il existe une représentation $\pi^{\prime}$ du groupe $S O\left(2 n-d_{\rho}\left(a+a_{-}\right)+1, F\right)$ et une inclusion

$$
\pi \hookrightarrow \rho\left\|^{(a-1) / 2} \times \cdots \times \rho\right\|^{\left(a_{-}+1\right) / 2} \times \pi^{\prime} .
$$

La conséquence de ce théorème est que l'on connaît très explicitement les modules de Jacquet des représentations dans $\Pi(\psi)$.

On considère la donnée endoscopique de $G$ dont le groupe $H$ est $S O\left(d_{\rho}\left(a+a_{-}\right)+1, F\right) \times S O\left(2 n-d_{\rho}(a+\right.$ $\left.\left.a_{-}\right)+1, F\right)$. Et pour $H$ on considère le morphisme $\psi_{1} \times \psi_{2}$ de $W_{F} \times S L(2, \mathbb{C})$ à valeurs dans $S p\left(d_{\rho}\left(a+a_{-}\right), \mathbb{C}\right) \times$ $S p\left(2 n-d_{\rho}\left(a+a_{-}\right), \mathbb{C}\right)$, où $\psi_{1}$ est la somme $\rho \otimes \sigma_{a} \oplus \rho \otimes \sigma_{a_{-}}$et $\psi_{2}$ est la somme des autres sous-représentations incluses dans $\psi$.

On a besoin de connaître le paquet $\Pi\left(\psi_{1}\right)$; on sait par 4.4 qu'il a 2 éléments si $a_{-} \neq 0$ et 1 élément sinon. La situation est donc particulièrement simple. On sait que l'élément de $I_{\text {cusp }}^{\text {st }}\left(\tilde{G L}\left(d_{\rho}\left(a+a_{-}\right), F\right)\right)$ qui définit ce paquet est l'image d'un prolongement à $\tilde{G L}\left(d_{\rho}\left(a+a_{-}\right), F\right)$ de la représentation tempérée $S t(\rho, a) \times S t\left(\rho, a_{-}\right)$. On écrit $S t(\rho, a) \times S t\left(\rho, a_{-}\right)$comme l'unique sous-module irréductible de l'induite :

$$
\rho||^{(a-1) / 2} \times \cdots \times\left.\rho\right|^{\left(a_{-}+1\right) / 2} \times S t\left(\rho, a_{-}\right) \times S t\left(\rho, a_{-}\right) \times \rho||^{-\left(a_{-}+1\right) / 2} \times \cdots \times\left.\rho\right|^{-(a-1) / 2} .
$$

Alors $\Pi\left(\psi_{1}\right)$ contient les sous-modules irréductibles de l'induite pour $S O\left(d_{\rho}\left(a+a_{-}\right)+1, F\right)$ :

$$
\left.\rho\right|^{(a-1) / 2} \times \cdots \times \rho \|^{\left(a_{-}+1\right) / 2} \times S t\left(\rho, a_{-}\right) .
$$

C'est un calcul de module de Jacquet expliqué en [13] 4.2 et analogue à ceux fait ici ; on montre en loc.cit. qu'un module de Jacquet convenable de la distribution stable dans $\Pi\left(\psi_{1}\right)$ (relativement au facteur $G L\left(d_{\rho}, F\right)$ ) est non nul et a pour transfert stable $S t\left(\rho, a_{-}\right) \times S t\left(\rho, a_{-}\right)$; avec les notations de loc.cit. c'est $J a c_{(a-1) / 2, \cdots,\left(a_{-}+1\right) / 2}$. Ce module de Jacquet est donc l'induite à $S O\left(2 a_{-}+1, F\right)$ de la représentation $S t\left(\rho, a_{-}\right)$. Si $a_{-}>0$, cette induite est de longueur 2 car la parité de $a_{-}$est la bonne (cf. 2) et $\Pi\left(\psi_{1}\right)$ contient nécessairement les 2 sous-modules de (1). Si $a_{-}=0,(1)$ a un unique sous-module irréductible nécessairement dans $\Pi\left(\psi_{1}\right)$. On a donc décrit $\Pi\left(\psi_{1}\right)$ comme l'ensemble des sous-modules irréductibles de (1) et quand il y en a 2, la distribution stable est la somme de ces 2 sous-modules puisqu'un module de Jacquet d'une distribution stable est stable (cf. [13]4.2)

On ne peut évidemment pas donner une description aussi précise de $\Pi\left(\psi_{2}\right)$ et de la distribution stable qui est formée avec ses éléments; on fixe donc des nombres complexes $c_{\pi_{2}}$ pour tout $\pi_{2} \in \Pi\left(\psi_{2}\right)$ tel que $\sum_{\pi_{2} \in \Pi\left(\psi_{2}\right)} c_{\pi_{2}} \pi_{2}$ soit stable. Ainsi la distribution stable correspondant à $\psi_{1} \times \psi_{2}$ est :

$$
\left(\sum_{\pi_{1} \in \Pi\left(\psi_{1}\right)} \pi_{1}\right) \otimes\left(\sum_{\pi_{2} \in \Pi\left(\psi_{2}\right)} c_{\pi_{2}} t r \pi_{2}\right) .
$$

Pour tout $\pi \in \Pi(\psi)$, on fixe $c_{\pi} \in \mathbb{C}$ tel que $\sum_{\pi \in \Pi(\psi)} c_{\pi} \pi$ soit stable. Et on pose $\epsilon_{a}(\pi):=\epsilon_{\mathcal{A}}(\pi)\left(z_{\rho \otimes \sigma_{a}}\right)$, où $z_{\rho \otimes \sigma_{a}}$ est l'élément non trivial de $Z_{\rho \otimes \sigma_{a}}$; on définit de même $\epsilon_{a_{-}}(\pi)$ si $a_{-} \neq 0$ sinon on pose $\epsilon_{a_{-}}(\pi)=1$. D'après [2] 
(30.14), la distribution (2) se transfère en une distribution $H$-stable de $G$ de la forme (à un scalaire près qui vient de l'imprécision dans le choix des $c_{\pi}$ )

$$
\sum_{\pi \in \Pi(\psi)} \epsilon_{a}(\pi) \epsilon_{a_{-}}(\pi) c_{\pi} \operatorname{tr} \pi
$$

Ecrivons explicitement ce transfert sur les caractères vus comme fonctions localement $L^{1}$ sur les éléments semisimples. Pour tout $\gamma_{G}$ élément semi-simple de $G$, on l'égalité où les $\Delta\left(\gamma_{G}, \gamma_{H}\right)$ sont les facteurs de transfert et où $\gamma_{H}$ parcourt un ensemble de représentant de classes de conjugaison dans $H$ dont la classe stable se transfère en celle de $\gamma_{G}$ :

$$
\begin{gathered}
\left(\sum_{\pi \in \Pi(\psi)} \epsilon_{a}(\pi) \epsilon_{a_{-}}(\pi) c_{\pi} \operatorname{tr} \pi\right)\left(\gamma_{G}\right)= \\
\sum_{\gamma_{H}} \Delta\left(\gamma_{G}, \gamma_{H}\right)\left(\left(\sum_{\pi_{1} \in \Pi\left(\psi_{1}\right)} \operatorname{tr} \pi_{1}\right)\left(\sum_{\pi_{2} \in \Pi\left(\psi_{2}\right)} c_{\pi_{2}} t r \pi_{2}\right)\right)\left(\gamma_{H}\right) .
\end{gathered}
$$

On va appliquer cette égalité en imposant à $\gamma_{G}$ d'être dans le sous-groupe de Levi $M \simeq G L\left(d_{\rho}, F\right) \times S O(2(n-$ $\left.\left.d_{\rho}\right)+1, F\right)$ de $G$. On écrit un tel élément sous la forme $\gamma_{G}=m \times \gamma^{\prime}$, où $m \in G L\left(d_{\rho}, F\right)$.

On note $M_{H}^{1}$ le sous-groupe de Levi de $H$ isomorphe à

$$
G L\left(d_{\rho}, F\right) \times S O\left(2\left(n_{1}-d_{\rho}\right)+1, F\right) \times S O\left(2 n_{2}, F\right)
$$

et $M_{H}^{2}$ celui qui est isomorphe à

$$
S O\left(2 n_{1}+1, F\right) \times G L\left(d_{\rho}, F\right) \times S O\left(2\left(n_{2}-d_{\rho}\right)+1, F\right) .
$$

Fixons $\gamma_{G} \in M$ comme ci-dessus mais on suppose que $m$ est elliptique dans $G L\left(d_{\rho}, F\right)$. Dans (4) il suffit alors de sommer sur les éléments $\gamma_{H}$ avec les propriétés imposées qui sont soit dans $M_{H}^{1}$ soit dans $M_{H}^{2}$. On note $Z_{M}$ le centre de $M$ et on applique cela non pas à $\gamma_{G}$ mais à l'ensemble des $\gamma_{G} z$ où $z$ parcourt $Z_{M}$. On prenant une limite convenable et on appliquant une formule de Casselman on peut remplacer dans (4), $\operatorname{tr} \pi$ par $\operatorname{tr} r e s_{M}(\pi)$ et les produits

$$
\sum_{\gamma_{H}} \Delta\left(\gamma_{G}, \gamma_{H}\right)\left(\operatorname{tr} \pi_{1} \operatorname{tr} \pi_{2}\right)\left(\gamma_{H}\right)
$$

par

$$
\sum_{\gamma_{H}^{1}} \Delta\left(\gamma_{G}, \gamma_{H}^{1}\right) \operatorname{tr}\left(r e s_{M_{H}^{1}} \pi_{1}\right) \operatorname{tr} \pi_{2}\left(\gamma_{H}^{1}\right)+\sum_{\gamma_{H}^{2}} \Delta\left(\gamma_{G}, \gamma_{H}^{2}\right) \operatorname{tr} \pi_{1} \operatorname{tr}\left(\operatorname{res}_{M_{H}^{2}} \pi_{2}\right)\left(\gamma_{H}^{2}\right),
$$

où $\gamma_{H}^{1}$ est dans $M_{H}^{1}$ et $\gamma_{H}^{2}$ est dans $M_{H}^{2}$.

En faisant varier $m$ dans l'ensemble des éléments elliptiques de $G L\left(d_{\rho}, F\right)$ on projette sur le caractère de la représentation cuspidale $\rho \|^{(a-1) / 2}$; parce que $\rho$ est cuspidale bien que l'on se limite aux éléments elliptiques, cela permet de faire disparaitre toutes les autres représentations c'est-à-dire remplacer les restrictions par les restrictions suivies par cette projection sur $\rho \|^{(a-1) / 2}$. Il faut vérifier que cette opération appliquée à n'importe quel élément de $\Pi\left(\psi_{2}\right)$ donne 0 . Cela résulte des propriétés standards des modules de Jacquet des paquets de séries discrètes que nous avons établies; pour que cette projection soit non nulle il faudrait que la sousreprésentation $\rho \otimes \sigma_{a}$ de $W_{F} \times S L(2, \mathbb{C})$ soit incluse dans $\psi_{2}$, ce qui n'est pas le cas par choix de $\psi_{2}$. Comme nous allons en avoir besoin ci-dessous remarquons que cette propriété reste vraie en remplaçant $(a-1) / 2$ par $\left(a^{\prime}-1\right) / 2$ pour tout $\left.\left.a^{\prime} \in\right] a_{-}, a\right]$ car $\rho \otimes \sigma_{a^{\prime}}$ pour un tel $a^{\prime}$ n'est pas sous représentation de $\psi$ par minimalité de $a_{-}$. Si $a>a_{-}+2$, on recommence ces opérations pour le Levi $M^{\prime}=G L\left(d_{\rho}, F\right) \times S O\left(2\left(n-2 d_{\rho}\right)+1, F\right)$ en projetant cette fois sur $\left.\rho\right|^{(a-3) / 2}$. On note

$$
M_{a, a_{-}} \simeq G L\left(d_{\rho}, F\right) \times \cdots \times G L\left(d_{\rho}, F\right) \times S O\left(2 n-d_{\rho}\left(a-a_{-}\right)+1, F\right)
$$

le sous-groupe de Levi de $G$ isomorphe où il y a $\left(a-a_{-}\right) / 2$ copies de $G L\left(d_{\rho}, F\right)$. Et on note

$$
M_{a, a_{-}}^{1}=G l\left(d_{\rho}, F\right) \times \cdots \times G L\left(d_{\rho}, F\right) \times S O\left(2 n_{1}-d_{\rho}\left(a-a_{-}\right)+1, F\right) \times S O\left(2 n_{2}+1, F\right)
$$

le sous-groupe de Levi de $H$ où il y a $\left(a-a_{-}\right) / 2$ copies de $G L\left(d_{\rho}, F\right)$.

Il faut maintenant comparer les facteurs de transfert : on a $\gamma_{G}=\times_{i \in\left[1,\left(a-a_{-}\right) / 2\right.} m_{i} \times \gamma^{\prime}$ et $\gamma_{H}={ }_{i \in\left[1,\left(a-a_{-}\right) / 2\right.}$ $m_{i} \times \gamma_{1}^{\prime} \times \gamma_{2}$ avec chaque $m_{i}$ dans un groupe $G L\left(d_{\rho}, F\right), \gamma^{\prime} \in S O\left(2 n-d_{\rho}\left(a-a_{-}\right)+1, F\right), \gamma_{1}^{\prime} \in S O\left(2 n_{1}-d_{\rho}(a-\right.$ $\left.\left.a_{-}\right)+1, F\right)$ et $\gamma_{2} \in S O\left(2 n_{2}+1, F\right)$. Et on est dans la situation où la classe stable de $\gamma_{1}^{\prime} \times \gamma_{2}$ correspond à la classe stable de $\gamma^{\prime}$ dans l'endoscopie pour $S O\left(2 n-d_{\rho}\left(a-a_{-}\right)+1, F\right)$. On vérifie que

$$
\Delta\left(\gamma_{G}, \gamma_{H}\right)=\Delta\left(\gamma^{\prime}, \gamma_{1}^{\prime} \gamma_{2}\right)
$$


le premier facteur de transfert étant pour $S O(2 n+1, F)$ et le second pour $S O\left(2 n-d_{\rho}\left(a-a_{-}\right)+1, F\right)$; cela résulte du calcul des intégrales orbitales pour les éléments d'un Levi.

Finalement on établit que la distribution sur $M_{a, a_{-}}$

$$
\sum_{\pi \in \Pi(\psi)} \epsilon_{a}(\pi) \epsilon_{a_{-}}(\pi) c_{\pi} \operatorname{proj}_{\rho||^{\left(a_{-}+1\right) / 2}} \cdots \operatorname{proj}_{\rho||^{(a-1) / 2}} \operatorname{res}_{M_{a, a_{-}}} \pi
$$

est un transfert de la distribution stable sur $M_{a, a_{-}}^{1}$ :

$$
\left(\sum_{\pi_{1} \in \Pi\left(\psi_{1}\right)} \operatorname{proj}_{\rho||^{(a-+1) / 2}} \cdots \operatorname{proj}_{\rho||^{(a-1) / 2}} \operatorname{res}_{M_{a, a_{-}}^{1}} \pi_{1}\right)\left(\sum_{\pi_{2} \in \Pi\left(\psi_{2}\right)} c_{\pi_{2}} \operatorname{tr} \pi_{2}\right) .
$$

Le résultat principal de 11] décrit exactement les termes $\operatorname{proj}_{\rho||^{\left(a_{-}+1\right) / 2}} \cdots \operatorname{proj}_{\rho||(a-1) / 2} \operatorname{res}_{M_{a, a_{-}}} \pi$ quand $\pi$ parcourt $\Pi(\psi)$. Exactement cette trace est 0 pour la moitié des représentations précisément celles pour lesquelles notre caractère $\epsilon_{\pi}$ associé à tout élément de $\Pi(\psi)$ [10], [11] par vérifie $\epsilon_{\pi}(\rho, a) \neq \epsilon_{\pi}\left(\rho, a_{-}\right)$et pour les autres est une représentation irréductible de la forme

$$
\left.\rho||^{(a-1) / 2} \otimes \cdots \otimes \rho\right|^{\left(a_{-}+1\right) / 2} \otimes \pi^{\prime}
$$

où $\pi^{\prime}$ est l'un des 2 sous-modules irréductibles de l'induite $S t\left(\rho, a_{-}\right) \times \pi^{\prime \prime}$, avec $\pi^{\prime \prime}$ est un élément de $\Pi\left(\psi_{2}\right)$; si $a_{-}=0$, il n'y a pas d'induite et on obtient directement $\pi^{\prime \prime}$. On note simplement $J_{a c}(a-1) / 2, \cdots,\left(a_{-}+1\right) / 2 \pi$ cette représentation irréductible. On a décrit $\Pi\left(\psi_{1}\right)$ et avec cela on vérifie que :

$$
\left(\sum_{\pi_{1} \in \Pi\left(\psi_{1}\right)} \operatorname{proj}_{\rho||^{\left(a_{-}+1\right) / 2}} \cdots \operatorname{proj}_{\rho||^{(a-1) / 2}} \operatorname{res}_{M_{a, a_{-}}^{1}} \pi_{1}\right)
$$

est $\otimes_{j \in\left[(a-1) / 2,\left(a_{-}+1\right) / 2\right]} \rho||^{j} \otimes i n d S t\left(\rho, a_{-}\right)$où l'induite est pour le groupe $S O\left(2 a_{-}+1, F\right)$ à partir du parabolique de Levi $G L\left(a_{-}, F\right)$. Ainsi la distribution pour $S O\left(2 n-d_{\rho}\left(a-a_{-}\right)+1, F\right)$

$$
\sum_{\pi \in \Pi(\psi)} \epsilon_{a}(\pi) \epsilon_{a_{-}}(\pi) c_{\pi} J a c_{(a-1) / 2, \cdots,\left(a_{-}+1\right) / 2} \pi
$$

est un transfert de la distribution (rappelons que $\left.n_{1}=\left(a+a_{-}\right) / 2\right)$ :

$$
\left(i n d_{G L\left(a_{-} d_{\rho}, F\right)}^{S O\left(2 a_{-}+1, F\right)} S t\left(\rho, a_{-}\right)\right) \otimes\left(\sum_{\pi_{2} \in \Pi\left(\psi_{2}\right)} c_{\pi_{2}} \pi_{2}\right)
$$

Si $a_{-}=0$, on peut tout de suite conclure : les groupes pour (5) et (6) sont les mêmes et le transfert est l'identité. Donc en particulier les coefficients $c_{\pi_{2}}$ et $c_{\pi}$ étant positifs on doit aussi avoir $\epsilon_{a}(\pi)=1$ si $J a c_{(a-1) / 2, \cdots, 1 / 2} \pi \neq 0$. De plus comme on l'a rappelé, la moitié des éléments de $\Pi(\psi)$ ont cette propriété de non nullité. On calcule le cardinal du groupe des caractères de $\operatorname{Cent}_{S p(2 n, \mathbb{C})}(\psi) / \operatorname{Cent}(\operatorname{Sp}(2 n, \mathbb{C}))$ triviaux sur $Z_{\rho \otimes \sigma_{a}}$; ce groupe est facilement mis en bijection avec le groupe des caractères de $\operatorname{Cent}_{S p\left(2 n-a d_{\rho}, \mathbb{C}\right)}\left(\psi_{2}\right) / \operatorname{Cent}(\operatorname{Sp}(2 n-$ $\left.\left.a d_{\rho}, \mathbb{C}\right)\right)$. Le cardinal de ce groupe est donc $2^{\ell_{\psi_{2}}-1}$ ce qui est la moitié du cardinal de $\Pi(\psi)$. Comme on a déjà montré que l'application $\pi \in \Pi(\psi)$ associe $\epsilon_{\mathcal{A}}$ est une bijection sur l'ensemble des caractères du centralisateur de $\psi$ triviaux sur le centre de $S p(2 n, \mathbb{C})\left(\mathrm{cf}\right.$.4.4), on voit que la propriété $\epsilon_{a}(\pi)=1$ caractérise les représentations $\pi$ de $\Pi(\psi)$ telles que $J a c_{(a-1) / 2, \cdots, 1 / 2} \pi \neq 0$ ce qui est l'énoncé cherché dans ce cas.

Supposons maintenant que $a_{-}>0$. Soient $\pi, \pi^{\prime} \in \Pi(\psi)$ et $\pi_{2} \in \Pi\left(\psi_{2}\right)$ tels que l'on ait l'égalité :

$$
\operatorname{Jac}_{(a-1) / 2, \cdots,\left(a_{-}+1\right) / 2} \pi \oplus J a c_{(a-1) / 2, \cdots,\left(a_{-}+1\right) / 2} \pi^{\prime}=S t\left(\rho, a_{-}\right) \times \pi_{2} .
$$

On montre d'abord que $c_{\pi}=c_{\pi^{\prime}}=c_{\pi_{2}}$; c'est un problème de transfert stable. D'une part on sait que $\sum_{\pi \in \Pi(\psi)} c_{\pi} \pi$ est le transfert d'un prolongement à $\tilde{G L}(2 n, F)$ de la représentation tempérée $\pi(\psi)$ de $G L(2 n, F)$ associée à $\psi$. D'autre part on sait que $\sum_{\pi_{2} \in \Pi\left(\psi_{2}\right)} c_{\pi_{2}} \pi_{2}$ est le transfert stable d'un prolongement à $\tilde{G L}\left(2 n_{2}, F\right)$ de la représentation tempérée $\pi\left(\psi_{2}\right)$ de $G L\left(2 n_{2}, F\right)$ associée à $\psi_{2}$. On sait que $\pi(\psi)$ est l'unique sous-module irréductible de l'induite

$$
\left.\theta\right|^{(a-1) / 2} \times \cdots \times\left.\rho\right|^{\left(a_{-}+1\right) / 2} \times S t\left(\rho, a_{-}\right) \times \pi\left(\psi_{2}\right) \times S t\left(\rho, a_{-}\right) \times\left.\rho\right|^{-\left(a_{-}+1\right) / 2} \times \cdots \times\left.\rho\right|^{-(a-1) / 2} .
$$


Donc on passe de $\pi(\psi)$ à $S t\left(\rho, a_{-}\right) \times \pi\left(\psi_{2}\right) \times S t\left(\rho, a_{-}\right)$en prenant des modules de Jacquet (cf. [13] 4.2) et on passe de $\pi\left(\psi_{2}\right)$ à la même représentation en induisant. Cela donne immédiatement l'égalité

$$
\sum_{\pi \in \Pi(\psi)} c_{\pi} J a c_{(a-1) / 2, \cdots,\left(a_{-}-1\right) / 2} \pi=\sum_{\pi_{2} \in \Pi\left(\psi_{2}\right)} c_{\pi_{2}}\left(S t\left(\rho, a_{-}\right) \times \pi_{2}\right) .
$$

On en déduit l'assertion en décomposant chaque représentation comme déjà expliqué. Pour $\pi_{2} \in \Pi\left(\psi_{2}\right)$, on note $\Pi\left(\pi_{2}\right)$ les 2 représentations, $\pi^{\prime}, \pi^{\prime \prime}$ de $\Pi(\psi)$ telles que

$$
\operatorname{Jac}_{(a-1) / 2, \cdots,\left(a_{-}+1\right) / 2}\left(\pi^{\prime} \oplus \pi^{\prime \prime}\right)=S t\left(\rho, a_{-}\right) \times \pi_{2} .
$$

On a donc sur $S O\left(2 n_{2}+2 d_{\rho} a_{-}+1, F\right)$ la distribution :

$$
\sum_{\pi_{2} \in \Pi\left(\psi_{2}\right)} c_{\pi_{2}} \sum_{\pi \in \Pi\left(\pi_{2}\right)} \epsilon_{a}(\pi) \epsilon_{a_{-}}(\pi) J a c_{(a-1) / 2, \cdots,\left(a_{-}+1\right) / 2} \pi
$$

qui est un transfert de la distribution sur $S O\left(2 d_{\rho} a_{-}+1, F\right) \times S O\left(2 n_{2}+1, F\right)$ :

$$
\left(i n d_{G L\left(d_{\rho} a_{-}, F\right)}^{S O\left(2 d_{\rho} a_{-}+F\right)} S t\left(\rho, a_{-}\right)\right)\left(\sum_{\pi_{2}} c_{\pi_{2}} \pi_{2}\right) .
$$

On écrit encore l'égalité qui en résute pour tout élément $\gamma \in G L\left(d_{\rho} a_{-}\right) \times S O\left(2 n_{2}+1, F\right)$ dont la partie dans $G L\left(d_{\rho} a_{-}\right)$est elliptique. Ensuite on projette sur le sous-espace vectoriel de $I_{\text {cusp }}\left(G L\left(d_{\rho} a_{-}\right)\right)$image de $S t\left(\rho, a_{-}\right)$. On vérifie aisément que les éléments de $\Pi\left(\psi_{2}\right)$ n'ont pas de module de Jacquet qui peuvent contribuer à une telle projection. Le facteur de transfert $\Delta(\gamma, \gamma)$, où $\gamma$ est d'abord vu comme un élément de $S O\left(2 n_{2}+2 d_{\rho} a_{-}+1, F\right)$ puis du groupe endoscopique $S O\left(2 d_{\rho} a_{-}+1, F\right) \times S O\left(2 n_{2}+1, F\right)$ vaut 1 .

On obtient alors une égalité :

$$
\begin{gathered}
\sum_{\pi_{2} \in \Pi\left(\psi_{2}\right)} c_{\pi_{2}}\left(\sum_{\pi \in \Pi\left(\pi_{2}\right)}\left(\epsilon_{a}(\pi) \epsilon_{a_{-}}(\pi)\right) \pi_{2}=\right. \\
2 \times\left(\sum_{\pi_{2} \in \Pi\left(\psi_{2}\right)} c_{\left.\pi_{2}\right)} \pi_{2}\right) .
\end{gathered}
$$

D'où $\epsilon_{a} \epsilon_{a_{-}}(\pi)=1$ pour tout $\pi_{2}$ et tout $\pi \in \Pi\left(\pi_{2}\right)$. Ensuite on conclut comme dans le cas où $a_{-}=0$; la moitié des éléments, $\pi$, de $\Pi(\psi)$ vérifie à la fois $J a c_{(a-1) / 2, \cdots,\left(a_{-}+1\right) / 2} \pi \neq 0$ et $\epsilon_{a}(\pi) \epsilon_{a_{-}}(\pi)=1$. Mais chacune des 2 conditions est satisfaite par exactement la moitié des éléments de $\Pi(\psi)$, elles sont donc équivalentes. C'est l'assertion cherchée.

\subsection{Description des paramètres des représentations cuspidales}

Théorème. L'ensemble des représentations cuspidales de $S O(2 n+1, F)$ est en bijection avec l'ensemble des couples $(\psi, \epsilon)$ où $\psi$ est un morphisme sans trou de $W_{F} \times S L(2, \mathbb{C})$ dans $S p(2 n, \mathbb{C})$ et $\epsilon$ est un caractère alterné $d u$ centralisateur de $\psi$ de restriction trivial au centre de $S p(2 n, \mathbb{C}$ ) (on renvoit à l'introduction pour une précision sur les notations).

Soit $\pi$ une représentation cuspidal et $\psi$ le morphisme tel que $\pi \in \Pi(\psi)$; on a déjà vérifié que $\psi$ est sans trou ; on note $\epsilon_{\mathcal{A}}(\pi)$ le caractère associé par Arthur à $\pi$; il est alterné par 4.5. Réciproquement soit $\psi$ un morphisme sans trou et $\pi \in \Pi(\psi)$. Soit $\rho$ une représentation cuspidale irréductible et unitaire d'un $G L\left(d_{\rho}, F\right), x \in \mathbb{R}$ et $\sigma$ une représentation irréductible de $S O\left(2 n-d_{\rho}+1, F\right)$ tel que

$$
\left.\pi \hookrightarrow \rho\right|^{x} \times \sigma .
$$

Le fait que $\pi$ soit cuspidal est exactement équivalent à ce qu'il ne soit pas possible de trouver de telles données satisfaisant (1). Supposons que $\pi$ n'est pas cuspidal et montrons que $\epsilon_{\mathcal{A}} \pi$ n'est pas alterné. Cela suffira. On fixe donc $\rho, x$ satisfaisant (1); $\sigma$ ne joue pas de rôle. On sait a priori que $x$ est un demi-entier (cf. 1.5) et d'après [10] par exemple qu'en notant encore $\rho$ la représentation irréductible de $W_{F}$ correspondant à $\rho$ la représentation définie par $\psi$ de $W_{F} \times S L(2, \mathbb{C})$ contient comme sous-représentation la représentation $\rho \otimes \sigma_{2 x+1}$; de plus comme $\pi$ est une série discrète, on a sûrement $x>0$. On écrit $a=2 x+1$ et $a \geq 2$. Comme $\psi$ est sans trou, $a_{-}$est défini et vaut $a-2$. Il suffit d'appliquer 4.5 pour voir que $\epsilon_{\mathcal{A}}(\pi)$ n'est pas alterné. 


\subsection{Au sujet de la combinaison linéaire stable dans un paquet.}

Soit encore $\psi$ un morphisme $\theta$-stable et $\theta$ discret de $W_{F} \times S L(2, \mathbb{C})$ dans $S p(2 n, \mathbb{C})$. On note $\sum_{\pi \in \Pi(\psi)} c_{\pi} \pi$ la distribution stable engendrée par les éléments du paquet $\Pi(\psi)$ avec $c_{\pi}$ des réels positifs ; pour fixer vraiment les $c_{\pi}$ on demande à cette distribution d'être le transfert de la trace de la représentation tempérée $\pi(\psi)$ de $G L(2 n, F)$ prolongé à $\tilde{G L}(2 n, F)$. On remarque que $\psi$ est uniquement déterminé par $\pi$ et que $c_{\pi}$ est donc aussi uniquement déterminé par $\pi$.

Il est naturel de conjecturer que les $c_{\pi}$ sont tous égaux à 1. Ceci est démontré dans [20] si la restriction de $\psi$ à $W_{F}$ est triviale sur le groupe de ramification modérée. Par changement de base, on devrait pouvoir étendre le résultat de Waldspurger à tous les morphismes tels que la restriction de $\psi$ à $W_{F}$ se factorise par le groupe de Weil d'une extension résoluble de $F$. Mais dans l'état actuel, il semble difficile d'aller au delà et la remarque suivante est un substitut qui permettra d'écrire les distributions stables (et endoscopiques) pour tous les paquets pas seulement ceux qui sont tempérés. Pour cela, il faut rappeler ce qu'est le support cuspidal partiel d'une représentation irréductible, $\pi$ d'un groupe classique, ici $S O(2 n+1, F)$ : c'est l'unique représentation cuspidale, $\pi_{\text {cusp }}$, d'un groupe de la forme $S O\left(2 n_{\text {cusp }}+1, F\right)$ (ce qui définit l'entier $n_{\text {cusp }}$ ) tel que $\pi$ soit un sous-quotient irréductible d'une induite de la forme $\sigma \times \pi_{c u s p}$ où $\sigma$ est une représentation irréductible de $G L\left(n-n_{c u s p}, F\right)$. Comme une représentation cuspidale, comme $\pi_{c u s p}$, est dans un unique paquet de série discrète, on a bien défini $c_{\pi_{\text {cusp }}}$ tout comme on a défini $c_{\pi}$ ci-dessus.

Remarque. Soit $\pi$ une série discrète dont on note $\pi_{c u s p}$ le support cuspidal partiel, alors $c_{\pi}=c_{\pi_{c u s p}}$.

On le démontre par récurrence sur $n$ et on réutilise la démonstration de 4.5. Dans la preuve de 4.5 on a déjà calculé $c_{\pi}$ en fonction d'une série discrète d'un groupe $S O\left(2 n^{\prime}+1, F\right)$ avec $n^{\prime}<n$ sous l'hypothèse que la représentation définie par $\psi$ contient une sous-représentation $\rho \otimes \sigma_{a}$ avec $a_{-}$défini et $\pi$ vérifiant $\epsilon_{a}(\pi)=\epsilon_{a_{-}}(\pi)$ avec les notations de cette preuve. Il est immédiat de voir que $\pi_{c u s p}$ est aussi le support cuspidal partiel de la représentation $\pi_{2}$ de $S O\left(2 n_{2}+1, F\right)$ pour laquelle on a montré que $c_{\pi}=c_{\pi_{2}}$. D'où le résultat par récurrence dans ce cas. On est donc ainsi ramené au cas où $\epsilon_{\mathcal{A}}(\pi)$ (le caractère associé par [2] 30.1 à $\pi$ ) est alterné. Faisons cette hypothèse sur $\epsilon_{\mathcal{A}}(\pi)$. D'après 4.4 et la description des paramétres des représentations cuspidales (4.6), $\pi$ est cuspidal si et seulement si $\psi$ est sans trou; il n'y a donc rien à démontrer dans le cas où $\psi$ est sans trou.

Il faut donc voir encore le cas où $\psi$ a des trous, ou encore, le cas où il existe $\rho \otimes \sigma_{a}$ une sous-représentation irréductible de $\psi$ avec $a>2$ telle que $\rho \otimes \sigma_{a-2}$ ne soit pas une sous-représentation de $\psi$. On fixe une telle représentation $\rho \otimes \sigma_{a}$ et on note $d_{\rho}$ la dimension de la représentation $\rho$ et on notera aussi $\rho$ la représentation cuspidale de $G L\left(d_{\rho}, F\right)$ associée à $\rho$ par la correspondance locale de Langlands ([6], []] $)$. On note $\psi^{\prime}$ le morphisme de $W_{F} \times S L(2, \mathbb{C})$ dans $S p\left(2\left(n-d_{\rho}\right), \mathbb{C}\right)$ qui est la somme des sous-représentations irréductibles incluses dans $\psi$ sauf $\rho \otimes \sigma_{a}$ qui est remplacée par $\rho \otimes \sigma_{a-2}$. On a montré en 10 et 11] (cf. l'introduction de 11]) que l'application $J a c_{(a-1) / 2}$ établit une bijection entre $\Pi(\psi)$ et $\Pi\left(\psi^{\prime}\right)$; rappelons ce qu'est cette application. Soit $\pi \in \Pi(\psi)$, alors il existe une unique représentation irréductible $\pi^{\prime}$ de $S O\left(2\left(n-d_{\rho}\right)+1, F\right)$ telle que $\pi$ soit un sous-module de l'induite $\left.\rho\right|^{(a-1) / 2} \times \pi^{\prime}$. Et on a montré que $\pi^{\prime} \in \Pi\left(\psi^{\prime}\right)$ et que tous les éléments de $\Pi\left(\psi^{\prime}\right)$ sont obtenus ainsi exactement une fois.

Par la compatibilité du transfert stable à la prise de module de Jacquet (13 4.2), on obtient le fait que $\sum_{\pi \in \Pi(\psi)} c_{\pi} J a c_{(a-1) / 2} \pi$ est un transfert d'un prolongement (bien déterminé) de la représentation tempérée $\pi\left(\psi^{\prime}\right)$ de $G L\left(2\left(n-d_{\rho}\right), F\right)$ à $\tilde{G L}\left(2\left(n-d_{\rho}\right), F\right)$. D'où l'égalité $c_{\pi}=c_{J a c_{(a-1) / 2} \pi}$. Et on applique l'hypothèse de récurrence à $J a c_{(a-1) / 2} \pi$. On obtient l'égalité $c_{\pi}=c_{\pi_{c u s p}}$ puisqu'il est bien clair que le support cuspidal partiel de $\pi$ est le même que celui de $\operatorname{Jac}_{(a-1) / 2} \pi$. Cela termine la preuve.

\section{$5 \quad$ Le cas des groupes orthogonaux impairs non déployés}

Ici $G(n)$ est un groupe orthogonal d'un espace de dimension $2 n+1$ d'une forme orthogonale ayant un noyau anisotrope de dimension 3. Les preuves ci-dessous n'ont rien d'originales et s'appliquent beaucoup plus généralement pour passer d'un groupe quasidéployé à une forme intérieure.

Et on note $G_{d}(n)$ la forme déployée de $S O(2 n+1, F)$. On peut encore définir comme en 1.3.2, l'espace $I_{\text {cusp }}^{\text {st }}(G(n))$. Soit $\pi$ une série discrète, l'argument donné en loc.cit. s'applique pour montrer que l'image du caractère de $\pi$ dans $I_{\text {cusp }}^{\text {st }}(G(n))$ est non nul. Il existe donc une distribution stable de la forme

$$
\sum_{\pi^{\prime} \in \mathcal{P}} c_{\pi^{\prime}} t r \pi^{\prime}
$$

où $\mathcal{P}$ est un ensemble de représentations elliptiques de $G(n)$ et $c_{\pi^{\prime}}$ sont des éléments de $\mathbb{C}$ et tel que $\pi \in \mathcal{P}$. 
D'après [3] 3.5 (un peu amelioré en [12] 4.5), le transfert induit un isomorphisme de $I_{\text {cusp }}^{\text {st }}(G(n))$ sur $I_{c u s p}^{\text {st }}\left(G_{d}(n)\right)$. Ainsi, il existe un ensemble $\mathcal{P}_{d}$ de représentations elliptiques de $G_{d}(n)$ et des nombres complexes, $c_{\pi_{d}^{\prime}}$ pour $\pi_{d}^{\prime} \in \mathcal{P}_{d}$ tels que

$$
\sum_{\pi_{d}^{\prime} \in \mathcal{P}_{d}} c_{\pi_{d}^{\prime}} \operatorname{tr} \pi_{d}^{\prime}
$$

soit un transfert stable de (1). Réciproquement, étant donné un paquet stable pour $G_{d}(n)$, c'est-à-dire une combinaison linéaire à coefficients complexes de caractères, stable, il existe une combinaison linéaire à coefficient complexes de caractères pour $G(n)$ qui est stable et qui se transfère en la combinaison de départ. Les caractères sont les caractères des représentations a priori elliptiques.

Ainsi pour $\pi$ une série discrète de $G(n)$, il existe au moins un morphisme $\psi$ de $W_{F} \times S L(2, \mathbb{C})$ dans $G^{*}(n)$ tel que $\pi$ soit dans un paquet stable se tranférant en le paquet $\Pi(\psi)$ formé de séries discrètes de $G_{d}(n)$. Ceci suppose évidemment les hypothèses déjà faites au sujet des lemmes fondamentaux. On dira encore que $\pi \in \Pi(\psi)$, $\Pi(\psi)$ étant maintenant vu comme un ensemble de représentations elliptiques de $G(n)$.

Théorème 1. Soit $\pi_{0}$ une représentation cuspidale de $G\left(n_{0}\right)$ et $\rho$ une représentation cuspidale autoduale irréductible d'un $G L\left(d_{\rho}, F\right)$. Soit $x_{0} \in \mathbb{R}$ tel que l'induite $\left.\rho\right|^{x_{0}} \times \pi_{0}$ soit réductible. Alors $x_{0} \in 1 / 2 \mathbb{Z}$.

On fixe $\pi_{0}$ et $x_{0}$ comme dans l'énoncé; si $x_{0}=0$, il n'y a rien à démontrer et on suppose donc que $x_{0}>0$. On note ici $\pi$ l'unique sous-module irréductible de $\left.\rho\right|^{x_{0}} \times \pi$. On reprend les notations, Jac $c_{x}$ de 1.5. On applique $J a c_{x_{0}}$ à $(1)$; soit $\pi^{\prime} \in \mathcal{P}$ tel que $J a c_{x_{0}} \pi^{\prime} \neq 0$ et contient $\pi_{0}$ dans sa décomposition en irréductible. Cela entraîne que $\pi^{\prime}$ est un sous-quotient irréductible de l'induite $\left.\rho\right|^{x_{0}} \times \pi_{0}$ et comme $\pi^{\prime}$ est elliptique cela nécessite que $\pi^{\prime}=\pi$. Ainsi $J a c_{x_{0}}$ appliqué à (1) est de la forme :

$$
c_{\pi} \pi_{0} \oplus \tau
$$

où $\tau$ dans (3) est une combinaison linéaire de représentations irréductibles dont aucun n'est équivalente à $\pi_{0}$. On vérifie que $J a c_{x_{0}}$ appliqué à (2) est un transfert de (3). En particulier, $J a c_{x_{0}}$ appliqué à (2) n'est pas nul. $\mathrm{Ou}$ encore, il existe une représentation elliptique de $G_{d}(n), \pi_{d}$, tel que $J a c_{x_{0}} \pi_{d} \neq 0$. Mais comme on sait que le support cuspidal des représentations elliptiques de $G_{d}(n)$ est comme celui des séries discrètes, demi-entier, on en déduit que $x_{0}$ est demi-entier. D'où le théorème.

On démontre de la même façon les théorèmes ci-dessous :

Théorème 2. Avec les hypothèses et notations du théorème précédent en particulier $\pi_{0}$ est une représentation cuspidale de $G(n)$. Soit $\psi_{0}$ un morphisme de $W_{F} \times S L(2, \mathbb{C})$ tel que $\pi_{0} \in \Pi\left(\psi_{0}\right)$. Alors $\psi_{0}$ est $\theta$-discret, sans trou et les points de réductibilité pour les induites de la forme $\rho \|^{x} \times \pi_{0}$ se calculent comme dans le cas déployé, c'est-à-dire que l'on a avec les notations de ce cas :

$$
x_{\rho, \pi_{0}}=\left(a_{\rho, \psi_{0}}+1\right) / 2 .
$$

Théorème 3. Soit $\pi$ une série discrète irréductible de $G(n)$ et soit $\psi$ un morphisme de $W_{F} \times S L(2, \mathbb{C})$ dans $S p(2 n, \mathbb{C})$ tel que $\pi \in \Pi(\psi)$. Alors $\psi$ est $\theta$-discret. De plus ce morphisme $\psi$ est uniquement déterminé par les blocs de Jordan de $\pi$ comme dans le cas déployé et correspond à celui qui a été associé à $\pi$ par [10] et [1]

Si $\psi$ n'est pas $\theta$-discret, comme dans le cas déployé toute représentation de $\Pi(\psi)$ est sous-module d'une induite convenable. Ceci est exclu pour $\pi$ qui est une série discrète, d'où le théorème 3 . La fin du théorème se démontre comme dans le cas déployé, nos références, [9], [10] et [1] ne font pas l'hypothèse que le groupe est déployé.

Pour terminer remarquons que l'on n'a pas ici calculé le nombre d'éléments d'un paquet $\Pi(\psi)$ et par voie de conséquence obtenu une paramétrisation des représentations cuspidales de $G(n)$; la méthode du cas déployé s'appuie sur les résultats de transfert endoscopique d'Arthur qu'il faudrait étendre. Il y a sans doute une méthode locale comme pour le transfert stable et cela vaudrait la peine de l'écrire en général.

\section{Références}

[1] Arthur J. : Unipotent automorphic representations : conjectures in Orbites unipotentes et représentations II, Astérisque 171-172, 1989, pp. 13-72 
[2] Arthur J. : An introduction to the trace formula prépublication

[3] Arthur J. : On local character relations, Selecta Math. 2, 1996, pp. 501-579

[4] Bernstein I. N., Zelevinsky A. V. : Induced Representations of Reductive p-adic groups 1 Ann de l'ENS, 10, 1977, pp. 147-185

[5] Clozel L. : On limit multiplicities of discrete series representations in spaces of automorphic forms, Inventiones math. 83, 1986, pp. 265-284

[6] Harris, M.; TAYlor, R. : The geometry and cohomology of some simple Shimura varieties,Annals of Math Studies, 151, Princeton Univ. Press, 2001

[7] Henniart, G. : Une preuve simple des conjectures de Langlands pour $G L_{n}$ sur un corps p-adique,Invent. Math., 139, 2000, pp. 439-455

[8] Lusztig G. : Classification of Unipotent Representations of Simple p-adic Groups, II,arXiv :math. $\mathrm{RT} / 0111248,2001$

[9] Megglin C. : Points de réductibilité pour les induites de cuspidales, à paraitre au Journal of Algebra 268, Number 1 (October 1, 2003 issue).

[10] Megalin C. : Classification des séries discrètes : paramètre de Langlands et exhaustivité,JEMS, 4, 143-200, 2002

[11] Megglin C., Tadic M. : Construction of discrete series for classical p-adic groups, journal de l'AMS, volume 15, 2002, pp 715-786

[12] Megglin C., Waldspurger J.-L. : Paquets stables de représentations tempérées et de rréduction unipotente pour $S O(2 n+1)$, Inventiones, 152, 461-623, 2003

[13] Megglin C., Waldspurger J.-L. : Sur le transfert des traces tordues d'un groupe linéaire à un groupe classique $p$-adique prépublication, http ://www.math.jussieu.fr/ moeglin

[14] Schneider M., Stuhler U. : Representation theory and sheaves on the Bruhat-Tits building Publ. Math. IHES 85, 1997, pp. 97-191

[15] Shahidi F. : Local coefficients and intertwining operators for GL(n), Compositio Math, 48, 1983, pp. $271-295$

[16] Silberger, A. : Special representations of reductive p-adic groups are not integrable,Ann. of Math., 111, 1980, pp. 571-587

[17] Waldspurger J.-L. : Transformation de Fourier et endoscopie, J. Lie Theory 10 (2000), pp. 195-206

[18] Waldspurger J.-L. : La formule de Plancherel d'après Harish-Chandra JIMJ, vol2, fasc 2, 2003

[19] Waldspurger J.-L. : Le groupe $G L_{N}$ tordu, sur un corps p-adique ,1e partie, prépublication 2005

[20] Waldspurger J.-L. : Le groupe $G L_{N}$ tordu, sur un corps p-adique ,2e partie, prépublication 2005

[21] Zelevinsky A. V. : Induced Representations of Reductive p-adic groups II Ann de l'ENS, 13, 1980, pp. $165-210$ 